\title{
Prior specification of neighbourhood and interaction structure in binary Markov random fields
}

\author{
Petter Arnesen and HÅkon TJelmeland \\ Department of Mathematical Sciences, Norwegian University of Science and Technology
}

\begin{abstract}
In this paper we propose a prior distribution for the clique set and dependence structure of binary Markov random fields (MRFs). In the formulation we allow both pairwise and higher order interactions. We construct the prior by first defining a prior for the neighbourhood system of the MRF, and conditioned on this we define a prior for the appearance of higher order interactions. Finally, for the parameter values we adopt a prior that allows for parameter values to equal, and in this way we reduce the effective number of free parameters. To sample from a resulting posterior distribution conditioned on an observed scene we construct a reversible jump Markov chain Monte Carlo (RJMCMC) algorithm. We circumvent evaluations of the intractable normalising constant of the MRF when running this algorithm by adopting a previously defined approximate auxiliary variable algorithm. We demonstrate the usefulness of our prior in two simulation examples and one real data example.
\end{abstract}

Key words: Auxiliary variables; Ising Model; Markov random fields; Reversible jump MCMC.

\section{Introduction}

MRFs are a well used model class in spatial statistics, see Kindermann and Snell (1980) and Hurn et al. (2003) for an introduction. In this paper we consider binary MRFs which is a subclass of discrete MRF. An MRF $x$ is usually defined by conditioning on a parameter vector $\phi$, so that we have $p(x \mid \phi)$. This $\phi$ models interactions between the components of $x$, and in applications these interactions are typically limited only to be pairwise, see for instance Besag (1974), Besag (1986), Friel and Rue (2007), Everitt (2012) and McGrory et al. (2012).

Mainly three arguments are made for constraining these models to include only pairwise interactions. Firstly, the interpretation of the parameters of higher order interaction can be difficult, for instance assigning values to the parameters in order to give the MRF some

Correponding author is Petter Arnesen, Department of Mathematical Sciences, Norwegian University of Science and Technology, Trondheim 7491, Norway. E-mail: petterar@math.ntnu.no. 
desired property can be a hard task. Secondly, if one allows for higher order interactions, the number of parameters grows exponentially as a function of the number of variables in a clique. And thirdly, because of the intractable normalising constant, binary and discrete MRFs are a computationally intensive class of models, especially if parameter inference is required for large dependency structures with higher order interactions. It has however been shown, see Descombes et al. (1995) and Tielmeland and Besag (1998), that for many data sets higher order interaction models outperforms pairwise models, for instance when an MRF is used as a prior distribution when recovering a noisy image. In those papers reasonable interpretation of higher order interactions are in fact given, and computations are carried out in reasonable time. In both the above papers, the number of parameters are reduced by manually setting the same value to groups of parameters. Only the second of the two papers are however performing parameter inference, which is made possible by approximating the maximal likelihood estimator obtained by applying a Markov chain Monte Carlo (MCMC) technique to estimate the normalising constant (Gever and Thompson 1992). Other, and more recent approximation techniques to circumvent the computational problems associated with discrete MRFs have also been proposed, see for instance Austad (2011), Everitt (2012) and McGrory et al. (2012). Note however that Austad (2011) is the only work of the three just mentioned which performs parameter inference for MRFs with higher order cliques. We discuss options for handling the normalising constant later in the paper.

When assigning a prior to the interaction structure of an MRF this can be done on three different levels. First one can assume the neighbourhoods and the parametric form of the MRF to be given, and assign a prior distribution for the values of the parameters only. This is for instance done in Heikkinen and Högmander (1994) and Rvdén and Titterington (1998). A second level of prior formulation is adopted in Arnesen and Tjelmeland (2015). The neighbourhoods are still fixed, but here priors are formulated for both the parametric form of the MRF and for the associated parameter values. An interpretational parametrisation inspired by Tjelmeland and Besag (1998) is used, and to reduce the effective number of free parameters without reducing the flexibility of the MRF one adopts a prior where groups of parameters are allowed to have exactly the same value. In this article we go to the third level of prior formulation by assigning a prior also for the neighbourhood system of the 
MRF, and to our knowledge this is the first attempt to learn the neighbourhood system of an MRF from observed data by such a fully Bayesian approach. As in Arnesen and Tjelmeland (2015) we keep the prior property that groups of parameters are allowed to be assigned the same parameter value, but we need to adopt a new parametrisation of the MRF. To obtain posterior samples we develop an RJMCMC sampling algorithm (Green 1995), and to cope with the intractable normalising constant we adopt the approximate exchange algorithm (Murray et al. 2006) of Everitt (2012).

Assigning a prior distribution to the dependence structure of $x$ is a much used approach in the theory of graphical modelling for categorical data, see Spiegelhalter et al. (1993), Madigan et al. (1995), Lauritzen (1996), Dellaportas and Forster (1999) and Massam et al. (2009), but the problems considered there are often small enough so that the normalising constant is easily calculated. Also, the variables in such problems often represent features of different nature, whereas we assume translational invariance of $x$ similar to all the other work on MRFs mentioned above. For graphical models this lack of invariance is compensated for by multiple observations of $x$, whereas we consider situations where only one scene $x$ is observed. For more details regarding the link between these two type of models the reader is referred to Arnesen and Tielmeland (2015).

The paper is organised as follows. In the next section, Section 2, we introduce notation and define some properties for the nodes in a rectangular lattice, while in Section 3 we assign binary random variables to these nodes and discuss two possible parametrisation of their joint probability distribution. In Section 4 we let this probability distribution be a stationary $\mathrm{MRF}$, and using both parametrisations we construct in Section 5 a prior distribution for the dependence structure and the parameter values of an MRF. In Section 6 we present the proposal distributions we use when sampling from the posterior distribution along with a discussion on how to handle the normalising constant. We investigate three examples in Section 7, and lastly we give some closing remarks in Section 8 . 


\section{Preliminaries}

In this section we consider a rectangular lattice with periodic boundary conditions and introduce the notation we need in later sections to define a prior for stationary MRFs on such a lattice.

Consider a rectangular $m \times n$ lattice and let $(i, j)$, where $i \in\{0,1, \ldots, m-1\}$ and $j \in$ $\{0,1, \ldots, n-1\}$, denote an arbitrary node in this lattice. Here we adopt the convention that $i$ and $j$ specify the vertical and horizontal positions of the node in the lattice, respectively, that $i=0$ is at the top of the lattice and $i=m-1$ at the bottom, and that $j=0$ and $j=n-1$ are at the left and right ends of the lattice, respectively. To denote sets of nodes we use lower case Greek letters, and in particular we denote the set of all nodes in the lattice by $\chi=\{(i, j) ; i=0, \ldots, m-1, j=0, \ldots, n-1\}$ and use $\lambda$ and $\lambda^{\star}$ to denote arbitrary subsets of $\chi$, i.e. $\lambda, \lambda^{\star} \subseteq \chi$. In much of what follows we assume the lattice to have torus boundary conditions.

Definition 1 If, for a rectangular lattice $\chi=\{(i, j) ; i=0, \ldots, m-1, j=0, \ldots, n-1\}$, the translation of a node $(i, j) \in \chi$ with an amount $(t, u) \in \chi$ is defined to be

$$
(i, j) \oplus(t, u)=(i+t \quad \bmod m, j+u \bmod n),
$$

we say that the lattice has torus boundary conditions.

The translation of each node in a set $\lambda \subseteq \chi$ by an amount $(t, u) \in \chi$ we denote by $\lambda \oplus(t, u)=$ $\{(i, j) \oplus(t, u) ;(i, j) \in \lambda\}$.

To denote sets of subsets of $\chi$ we use upper case Greek letters, and at the next level we denote sets of sets of subsets of $\chi$ by upper case Roman letters. In particular we let $\Omega(\chi)=\{\lambda ; \lambda \subseteq \chi\}$ denote the set of all subsets of $\chi$ and use $\Lambda, \Lambda^{\star} \subseteq \Omega(\chi)$ to denote arbitrary subsets of $\Omega(\chi)$. The $\Omega(\chi)$ is often called the power set of $\chi$, and one should note that in particular it includes the empty set and $\chi$ itself. We let $L$ denote the partition of $\Omega(\chi)$ we get by identifying all subsets of $\chi$ that are translations of each other.

Definition 2 Let $L$ be the partition of $\Omega(\chi)$ where, for any two distinct subsets of nodes $\lambda, \lambda^{\star} \subseteq \chi$, there exists a $\Lambda \in L$ so that $\left\{\lambda, \lambda^{\star}\right\} \subseteq \Lambda$ if and only if there exists $a(t, u) \in \chi$ so that $\lambda^{\star}=\lambda \oplus(t, u)$. 
The elements in the partition we call cells. One can note that two of the cells in $L$ consist of only one element each, namely $\{\emptyset\}$ and $\{\chi\}$, whereas all other cells have $m n$ elements. One example of a cell with $m n$ elements is $\{\{(i, j)\} ;(i, j) \in \chi\}$, and another example is $\{\{(i, j),(i, j) \oplus(1,0)\} ;(i, j) \in \chi\}$. One can also note that all elements in a cell $\Lambda \in L$ contains the same number of nodes, and we denote this number by $\tau(\Lambda)$. For instance we have $\tau(\{\{(i, j)\} ;(i, j) \in \chi\})=1$ and $\tau(\{\{(i, j),(i, j) \oplus(1,0)\} ;(i, j) \in \chi\})=2$.

As all elements in a cell $\Lambda \in L$ are translations of each other we may specify a cell by specifying the relative positions of the nodes in the elements in that cell. This suggests a more intuitive notation for the cells, where one represents each node in an element of a cell by a box and arranges the boxes according to the relative positions of these nodes. For instance we get $\square=\{\{(i, j)\} ;(i, j) \in \chi\}$, $\boldsymbol{\square}=\{\{(i, j),(i, j) \oplus(1,0)\} ;(i, j) \in \chi\}$, and $\boxplus=\{\{(i, j),(i, j) \oplus(1,-1),(i, j) \oplus(1,0)\} ;(i, j) \in \chi\}$. In the following we use this box representation to refer to specific cells in $L$, and use the $\Lambda \in L$ notation whenever we need to refer to a generic cell.

For two distinct cells $\Lambda, \Lambda^{\star} \in L$ we let $N\left(\Lambda, \Lambda^{\star}\right)$ denote the number of elements $\lambda^{\star} \in \Lambda^{\star}$ that is a strict subset of an (arbitrary) element $\lambda \in \Lambda$, i.e.

$$
N\left(\Lambda, \Lambda^{\star}\right)=\operatorname{card}\left(\left\{\lambda^{\star} \in \Lambda ; \lambda^{\star} \subset \lambda \text { for some } \lambda \in \Lambda\right\}\right)
$$

where $\operatorname{card}(A)$ denotes the cardinality, or the number of elements, in the set $A$. That this number is the same for all $\lambda \in \Lambda$ follows from the fact that $\lambda \in \Lambda \Leftrightarrow \lambda \oplus(t, u) \in \Lambda$ for any $(t, u) \in \chi$. Using the box representation of the cells in $L$ it is easy to see what $N\left(\Lambda, \Lambda^{\star}\right)$ becomes. We have for instance $N(\Theta, \square)=2, N(\boxplus, \square)=3, N(\boxplus, \boxminus)=1$ and $N(\square, \Theta)=0$. We use whether or not some elements in a cell $\Lambda^{\star} \in L$ is a subset of an element in another (arbitrary) cell in $\Lambda \in L$ to define a partial ordering of the cells in $L$.

Definition 3 For any $\Lambda, \Lambda^{\star} \in L$ we define

$$
\Lambda^{\star} \prec \Lambda \Leftrightarrow N\left(\Lambda, \Lambda^{\star}\right)>0 \text {. }
$$

For instance, we have $\square \prec \theta, \square \prec \boxplus$ and $\boxminus \prec \boxplus$, but $\square \nprec 日$ and $\boxminus \nprec \square$. Clearly we also have $\{\emptyset\} \prec \Lambda$ for any $\Lambda \in L \backslash\{\emptyset\}$. 


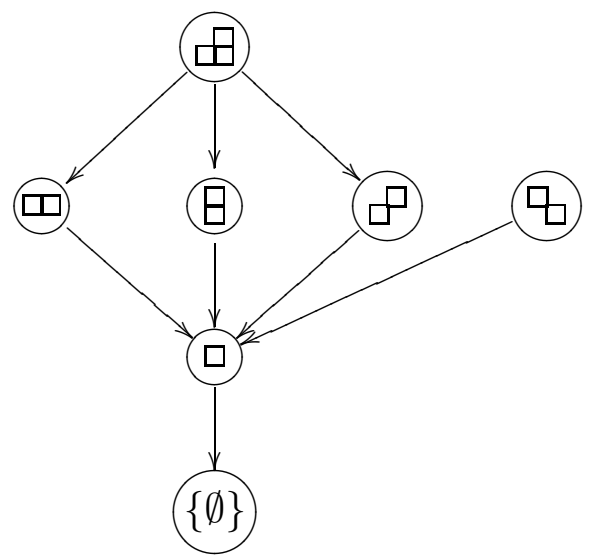

Figure 1: DAG representation of a dense set $M \subset L$.

In Section 4 we consider MRFs defined on the lattice $\chi$ and use a subset of the cells in $L$ to specify the set of cliques for the MRF. We denote such a subset by $M \subseteq L$, and use the term clique types for the elements in $M$. The cliques of the MRF will thereby be all $\lambda \in \Lambda$ for all $\Lambda \in M$, and will say that $\lambda \in \Lambda$ is of clique type $\Lambda$ for $\Lambda \in M$. We also say that a $\Lambda \in L$ is on if $\Lambda \in M$, and otherwise $\Lambda$ is off. We also define a prior for $M$ and then restrict the attention to sets $M \subseteq L$ that are dense.

Definition $4 A$ set of cells $M \subseteq L$ is said to be dense if $\left\{\Lambda^{\star} \in L ; \Lambda^{\star} \prec \Lambda\right\} \subseteq M$ for all $\Lambda \in M$.

We visualise the elements in a dense set $M \subseteq L$ as a directed acyclic graph (DAG), where the DAG has one vertex for each cell $\Lambda \in M$ and an edge from the vertex representing a $\Lambda^{\star} \in M$ to the vertex representing a $\Lambda \in M$ whenever $\Lambda^{\star} \prec \Lambda$ and $\tau\left(\Lambda^{\star}\right)=\tau(\Lambda)+1$. For instance,

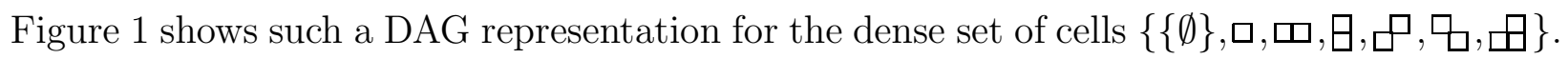
In the next section we present two parametrisations of binary stationary distributions defined on $\chi$.

\section{Binary stationary distributions on a torus}

Consider a rectangular lattice $\chi=\{(i, j) ; i=0, \ldots, m-1, j=0, \ldots, n-1\}$ with torus boundary conditions as in Section 2. Now, to each node $(i, j) \in \chi$ we associate a binary 
variable $x_{i, j} \in\{0,1\}$, and let $x=\left(x_{i, j} ;(i, j) \in \chi\right)$ denote a vector of all these binary variables. Associating the value 0 with white and the value 1 with black we can think of an $x$ as a colouring of the lattice $\chi$. A colouring where the nodes in a set $\lambda \subseteq \chi$ are black and all other nodes are white we denote by $\mathbf{1}^{\lambda}$, i.e. $\mathbf{1}^{\lambda}=\left(x_{i, j}=I((i, j) \in \lambda) ;(i, j) \in \chi\right)$, where $I(\cdot)$ is the indicator function that equals unity whenever the argument is true and zero otherwise.

We denote a distribution for $x$ by $p(x)$ and assume the positivity condition $p(x)>0$ for all $x \in\{0,1\}^{m n}$. Thus, we can write

$$
p(x)=Z \exp \{U(x)\}
$$

where $Z$ is a (typically computationally intractable) normalising constant and $-U(x)$ is often referred to as the energy function of $x$. In the following our focus is on stationary distributions $p(x)$.

Definition 5 A random vector $x$ defined on a rectangular lattice $\chi$ with torus boundary conditions is said to be stationary if and only if $p\left(\mathbf{1}^{\lambda}\right)=p\left(\mathbf{1}^{\lambda \oplus(t, u)}\right)$ for all $\lambda \in \Omega(\chi)$ and $(t, u) \in \chi$

In the remainder of this section we consider two representations of $U(x)$ and in particular discuss the consequences of imposing the stationarity assumption. In Section 4 we use each of these two representations as a basis to define a corresponding parametrisation for stationary binary MRFs. The first representation is defined using pseudo-Boolean function theory, while the second representation is defined directly on $U(x)$.

Following Tielmeland and Austad (2012), we note that $U(x)$ is a pseudo-Boolean function and thereby can be expressed as

$$
U(x)=\sum_{\lambda \in \Omega(\chi)} \beta^{\lambda} \prod_{(i, j) \in \lambda} x_{i, j}
$$

We refer to the $\beta$ parameters as interaction parameters. More details on pseudo-Boolean functions and their properties can be found in Hammer and Holzman (1992) and Grabisch et al. (2000). Using Definition 5 above, we state a theorem that identifies what restrictions the set $\left\{\beta^{\lambda} ; \lambda \in \Omega(\chi)\right\}$ must fulfil for $p(x)$ to be stationary. 
Theorem 1 A random vector $x$ defined on a rectangular lattice $\chi=\{(i, j) ; i=0, \ldots, m-$ $1, j=0, \ldots, n-1\}$ with torus boundary conditions is stationary if and only if $\beta^{\lambda}=\beta^{\lambda \oplus(t, u)}$ for all $\lambda \in \Omega(\chi)$ and $(t, u) \in \chi$. We then say that $\beta^{\lambda}$ is translational invariant.

The result in this theorem is a special case of Theorem 2 of Arnesen and Tielmeland (2015). The theorem above states that two interaction parameters $\beta^{\lambda}$ and $\beta^{\lambda^{\star}}$ must have the same value whenever $\lambda$ and $\lambda^{\star}$ belong to the same cell $\Lambda \in L$ in the partition of $\Omega(\chi)$ defined in Section 2. Thus, for $x$ to be stationary $\beta^{\lambda}$ must take the same value for all $\lambda \in \Lambda$ for each $\Lambda \in L$, and we denote the common value of $\beta^{\lambda}$, for all $\lambda \in \Lambda$, by $\beta^{\Lambda}$. Using the box notation introduced above we then in particular have that $\beta \square, \beta \boxminus$ and $\beta \boxplus$ are the common values for all $\beta^{\lambda}$ for $\lambda \in \square, \lambda \in \boxminus$ and $\lambda \in \boxminus$, respectively.

The second representation we consider for $U(x)$ is the quite naive one obtained by just representing the value of $U(x)$ for each possible vector $x$. For each $\lambda \in \Omega(\chi)$ we introduce a parameter $\phi^{\lambda}$ and write

$$
U\left(\mathbf{1}^{\lambda}\right)=\phi^{\lambda}, \lambda \in \Omega(\chi)
$$

The stationarity assumption puts similar restrictions on the $\phi$ parameters as it did for the $\beta$ parameters.

Theorem 2 A random vector $x$ defined on a rectangular lattice $\chi=\{(i, j) ; i=0, \ldots, m-$ $1, j=0, \ldots, n-1\}$ with torus boundary conditions is stationary if and only if $\phi^{\lambda}=\phi^{\lambda \oplus(t, u)}$ for all $\lambda \in \Omega(\chi)$ and $(t, u) \in \chi$. We then say that $\phi^{\lambda}$ is translational invariant.

Proof of Theorem 2 Combining (1) and (3) we get

$$
p\left(\mathbf{1}^{\lambda}\right)=Z \exp \left\{\phi^{\lambda}\right\}
$$

for all $\lambda \in \Omega(\chi)$. Thus we have that

$$
\phi^{\lambda}=\phi^{\lambda \oplus(t, u)} \Leftrightarrow p\left(\mathbf{1}^{\lambda}\right)=p\left(\mathbf{1}^{\lambda \oplus(t, u)}\right)
$$

for any $\lambda \in \Omega(\chi)$ and $(t, u) \in \chi$, and the result follows.

Corresponding to what we have for the $\beta$ parameters, we thus have that for $x$ to be stationary we must have that $\phi^{\lambda}$ must take the same value for all $\lambda \in \Lambda$ for each $\Lambda \in L$. We therefore 
also introduce a notation corresponding to what we did for the $\beta$ parameters, and denote the common value of $\phi^{\lambda}$ for all $\lambda \in \Lambda$ by $\phi^{\Lambda}$. In particular we then have that $\phi$ is the common value for all $\lambda \in \square$ and correspondingly $\phi \boxminus$ and $\phi \boxminus$ for all $\lambda \in \boxminus$ and all $\lambda \in \boxplus$, respectively. It should be noted that even though we use a similar notation for the $\beta$ and $\phi$ parameters, the correspondence is not as strong as our notation may suggest. For a given colouring $x$ of the nodes in $\chi$, the associated $\phi$ parameter is a function of all the components of $x$, whereas the associated $\beta$ parameter is only a function of a subset of the nodes. The next theorem states a one-to-one correspondence between $\left\{\beta^{\Lambda} ; \Lambda \in L\right\}$ and $\left\{\phi^{\Lambda} ; \Lambda \in L\right\}$.

Theorem 3 Consider a stationary random vector $x$ defined on a rectangular lattice $\chi=$ $\{(i, j) ; i=0, \ldots, m-1, j=0, \ldots, n-1\}$ with torus boundary conditions. Then there is a one-to-one correspondence between $\left\{\beta^{\Lambda} ; \Lambda \in L\right\}$ and $\left\{\phi^{\Lambda} ; \Lambda \in L\right\}$.

Proof of Theorem 3 From (2) and (3) we have that

$$
\phi^{\lambda}=\sum_{\lambda^{\star} \in \Omega(\chi)} \beta^{\lambda^{\star}} \prod_{(i, j) \in \lambda^{\star}} \mathbf{1}_{i, j}^{\lambda^{\star}}=\beta^{\lambda}+\sum_{\lambda^{\star} \subset \lambda} \beta^{\lambda^{\star}} \text { for all } \lambda \in \Omega(\chi) .
$$

By using Moebious inversion (Lauritzen 1996) it follows from this that

$$
\beta^{\lambda}=\phi^{\lambda}-\sum_{\lambda^{\star} \subset \lambda}(-1)^{\operatorname{card}(\lambda)-\operatorname{card}\left(\lambda^{\star}\right)-1} \phi^{\lambda^{\star}}
$$

If we invoke the translation invariance for both representations we get from (4) that

$$
\phi^{\Lambda}=\beta^{\Lambda}+\sum_{\Lambda^{\star} \prec \Lambda} N\left(\Lambda, \Lambda^{\star}\right) \beta^{\Lambda^{\star}}
$$

We see that we can use this expression to compute all $\left\{\phi^{\Lambda} ; \Lambda \in L\right\}$ from $\left\{\beta^{\Lambda} ; \Lambda \in L\right\}$ recursively. Firstly we can compute the $\phi^{\Lambda}$ for which $\tau(\Lambda)=0$, i.e. $\phi^{\{\emptyset\}}=\beta^{\{\emptyset\}}$. Next we can calculate the $\phi^{\Lambda}$ for which $\tau(\Lambda)=1$, thereafter all $\phi^{\Lambda}$ for which $\tau(\Lambda)=2$, and so forth until $\phi^{\Lambda}$ have been calculated for all $\Lambda \in L$. Thus, $\left\{\phi^{\Lambda} ; \Lambda \in L\right\}$ is uniquely specified by $\left\{\beta^{\Lambda} ; \Lambda \in L\right\}$.

If we again invoke the translation invariance for both representations we get from (5)

$$
\beta^{\Lambda}=\phi^{\Lambda}-\sum_{\Lambda^{\star} \prec \Lambda}(-1)^{\operatorname{card}(\Lambda)-\operatorname{card}\left(\Lambda^{\star}\right)-1} N\left(\Lambda, \Lambda^{\star}\right) \phi^{\Lambda^{\star}}
$$


where we correspondingly get that $\left\{\beta^{\Lambda} ; \Lambda \in L\right\}$ can be recursively computed from $\left\{\phi^{\Lambda} ; \Lambda \in L\right\}$. Again one must first compute $\beta^{\Lambda}$ for $\tau(\Lambda)=0$, i.e. $\beta^{\{\emptyset\}}$, then $\beta^{\Lambda}$ for which $\tau(\Lambda)=1$, thereafter all $\beta^{\Lambda}$ for which $\tau(\Lambda)=2$, and so forth. Thereby $\left\{\beta^{\Lambda} ; \Lambda \in L\right\}$ is also uniquely specified by $\left\{\phi^{\Lambda} ; \Lambda \in L\right\}$ and the proof is complete.

In the next section we present stationary binary MRFs, and use the two representations introduced above to establish a parametrisation of MRFs that is suitable for a fully Bayesian modelling.

\section{Binary MRFs}

In this section we first give a brief introduction to binary MRFs (Cressie 1993; Hurn et al. 2003). Thereafter we assume the MRF to be stationary and defined on a lattice with torus boundary conditions, and discuss what consequences the Markov assumption then has for the $\beta$ and $\phi$ representations introduced above. Finally we use each of the two representations to define corresponding parametrisations of an MRF.

Consider a rectangular lattice $\chi=\{(i, j) ; i=0, \ldots, m-1, j=0, \ldots, n-1\}$ as in Section 2 and a corresponding random vector $x=\left(x_{i, j} ;(i, j) \in \chi\right)$ where $x_{i, j} \in\{0,1\}$. In addition to the notation introduced above we let, for $\lambda \subseteq \chi, x_{\lambda}=\left(x_{i, j} ;(i, j) \in \lambda\right)$ denote the collection of random variables associated with the nodes in $\lambda$, and let $x_{-(i, j)}=x_{\chi \backslash\{(i, j)\}}$. A binary MRF is defined with respect to a so-called neighbourhood system $\Psi=\left\{\psi_{(i, j)} ;(i, j) \in \chi\right\}$ where $\psi_{(i, j)} \subseteq \chi \backslash\{(i, j)\}$ and $(i, j) \in \psi_{(t, u)} \Leftrightarrow(t, u) \in \psi_{(i, j)}$. The random vector $x$ is then said to be an MRF with respect to $\Psi$ if $p(x)>0$ for all $x \in\{0,1\}^{m n}$ and it fulfils the Markov property

$$
p\left(x_{i, j} \mid x_{-(i, j)}\right)=p\left(x_{i, j} \mid x_{\psi_{(i, j)}}\right)
$$

for all $(i, j) \in \chi$ and $x \in\{0,1\}^{n m}$. Given a neighbourhood system $\Psi$, a set of nodes $\lambda \subseteq \chi$ is said to be a clique if $(i, j) \in \psi_{(t, u)}$ for all distinct pair of nodes $(i, j),(t, u) \in \lambda$. In particular one can note that the empty set $\emptyset$ is always a clique, and so is $\{(i, j)\}$ for all $(i, j) \in \chi$. We denote the set of all cliques by $\Upsilon$, and let $\Upsilon_{\max }$ denote the set of all maximal cliques, where a clique is said to be maximal if it is not a subset of another clique. Clearly $\Upsilon \subseteq \Omega(\chi)$, 
$\Upsilon_{\max } \subset \Omega(\chi)$ and $\Upsilon_{\max } \subset \Upsilon$. The Hammersley-Clifford theorem (Clifford 1990) then states that $x$ is an MRF with respect to $\Psi$ if and only if $U(x)$ can be expressed as

$$
U(x)=\sum_{\lambda \in \Upsilon_{\max }} V_{\lambda}\left(x_{\lambda}\right),
$$

where $V_{\lambda}\left(x_{\lambda}\right)$ is referred to as the potential function for the maximal clique $\lambda \in \Upsilon_{\max }$. Combining (2) and (8) it follows that $\beta^{\lambda}=0$ for all $\lambda \notin \Upsilon$. Thus, $U(x)$ for an MRF is given by

$$
U(x)=\sum_{\lambda \in \Upsilon} \beta^{\lambda} \prod_{(i, j) \in \lambda} x_{i, j},
$$

see Tjelmeland and Austad (2012) for a formal proof.

In the following we assume $x$ to be defined on a lattice with torus boundary conditions, to be stationary, and to be an MRF with respect to a neighbourhood system $\Psi$. Theorem 1 then gives that $\beta^{\lambda}$ must be translational invariant, and in particular we must then have $\beta^{\lambda}=0 \Leftrightarrow \beta^{\lambda \oplus(t, u)}=0$ for $\lambda \in \Omega(\chi),(t, u) \in \chi$. Without loss of generality we can thereby assume also the clique system to be translational invariant in that

$$
\lambda \in \Upsilon \Leftrightarrow \lambda \oplus(t, u) \in \Upsilon \text { for all } \lambda \in \Omega(\chi),(t, u) \in \chi .
$$

In turn this implies that the neighbourhoods are translational invariant in that $\psi_{(i, j) \oplus(t, u)}=$ $\psi_{(i, j)} \oplus(t, u)$ for all $(i, j),(t, u) \in \chi$. From (10) it follows that we can find an $M \subseteq L$ so that $\Upsilon=\bigcup_{\Lambda \in M} \Lambda$, and again using that $\beta^{\lambda}$ is translational invariant we get that (9) can be reformulated as

$$
U(x)=\sum_{\Lambda \in M}\left[\beta^{\Lambda} \sum_{\lambda \in \Lambda} \prod_{(i, j) \in \lambda} x_{i, j}\right] .
$$

Thus, a stationary MRF defined on a lattice with torus boundary conditions is specified by the set $M$ and the values of the parameters $\left\{\beta^{\Lambda}, \Lambda \in M\right\}$. A natural and frequently used assumption in statistics is to allow higher-order interactions in a model only if corresponding lower-order interactions already are present. In the formulation of the MRF discussed above this means that if $\Lambda^{\star} \prec \Lambda$ we can allow $\Lambda \in M$ only if $\Lambda^{\star} \in M$, i.e. that $M$ is dense. In the following we therefore restrict $M$ to be dense. In addition we restrict, without loss of generality, that $\{\emptyset\}$ always is contained in $M$. 
Introducing for instance the constraint $\beta^{\{\emptyset\}}=0$, the linear independence of the products of $x$ in (11) makes the corresponding $p(x)$ an identifiable parametric model. In a fully Bayesian model formulation one would then need to put a prior on $M$ and the parameter set $\left\{\beta^{\Lambda}, \Lambda \in M \backslash\{\{\emptyset\}\}\right\}$. However, the $\beta$ parameters relate to cliques of different sizes and this makes the interpretation difficult. In particular we think the values of $\beta$ parameters associated with large cliques apriori should be expected to be closer to zero than values of parameters associated with smaller cliques, but we are not able to make this statement more precise than this. In the following we find an alternative parametrisation based on the $\phi$ representation discussed above and argue that this makes it easier to formulate a reasonable prior.

Given a stationary MRF defined on a lattice with torus boundary conditions specified by $M$ and $\left\{\beta^{\Lambda} ; \Lambda \in M\right\}$ and recalling that $\beta^{\Lambda}=0$ for $\Lambda \in L \backslash M$, we can use (6) to find the $\phi$ representation $\left\{\phi^{\Lambda} ; \Lambda \in L\right\}$ of the model. In contrast to the situation for the $\beta$ parameters, the Markov assumption does not induce any particular zero structure for the $\phi$ parameters. However, recalling that we have assumed $M$ to be dense, we easily see from (6) and (7) that there is a one-to-one correspondence between $\left\{\beta^{\Lambda} ; \Lambda \in M\right\}$ and $\left\{\phi^{\Lambda} ; \Lambda \in M\right\}$. An alternative to the $\beta$ parametrisation discussed above is therefore to parametrise the model by $M$ and $\left\{\phi^{\Lambda} ; \Lambda \in M\right\}$. One could note that then the remaining $\phi$ parameters, $\left\{\phi^{\Lambda} ; \Lambda \in L \backslash M\right\}$, are given by (6) and (7) as linear functions of $\left\{\phi^{\Lambda} ; \Lambda \in M\right\}$. From the definition of the $\phi$ parameters in (3) we see that they essentially just specify the probabilities for different realisations of $x$. If no specific prior information is available and suggests otherwise it therefore seems reasonable to assign a prior to $\left\{\phi^{\Lambda} ; \Lambda \in M\right\}$ where the various parameters are exchangeable.

\section{Prior distribution}

When constructing our prior distribution for the parameters in an MRF we have three properties in mind. Firstly, we want to assign a positive prior probability to the event that $\Lambda \in L$ is on or off. This is naturally done in the $\beta$ parametrisation, but Theorem 3 still enables us to work with the $\phi$ parameters. Secondly, we want a positive prior probability 
for the event that groups of clique types have exactly the same parameter value, and thirdly we want to assign a prior distribution to the parameter values of the MRF. As discussed in the last two paragraphs in the previous section, the two last aspects are most naturally formulated with the $\phi$ parametrisation. Thus, the main focus will be on the $\phi$ parametrisation for the reminder of this paper, and in the following we describe how we define a prior so that all the three properties discussed above are obtained.

For the first property discussed above we note that setting a $\Lambda \in L$ to be off is equivalent to setting

$$
\phi^{\Lambda}=\sum_{\Lambda^{\star} \prec \Lambda}(-1)^{\operatorname{card}(\Lambda)-\operatorname{card}\left(\Lambda^{\star}\right)-1} N\left(\Lambda, \Lambda^{\star}\right) \phi^{\Lambda^{\star}},
$$

by (7). Recalling that $L$ is required to be dense, we have that if $\Lambda$ is on, all $\Lambda^{\star} \in L$ where $\Lambda^{\star} \prec \Lambda$ must be on as well. As in the previous section we let $M \subseteq L$ denote the set of all clique types in $L$ that is on. Moreover, for integers $k \geq 0$ we let $M_{k}=\{\Lambda \in M ; \tau(\Lambda)=k\}$ be the set of all $k$ 'th order clique types in $M$. As mentioned above we restrict $M$ always to contain $\{\emptyset\}$, so clearly $M_{0}=\{\{\emptyset\}\}$ and one must have $M_{1}=\emptyset$ or $M_{1}=\{\square\}$. Moreover, one should note that $M_{2}$ is one-to-one with the neighbourhood system $\Psi$, and because of symmetry in the neighbourhoods $\operatorname{card}\left(\psi_{(i, j)}\right)=2 \cdot \operatorname{card}\left(M_{2}\right)$ for all $(i, j) \in \chi$. We assume the prior distribution for $M, p(M)$, to have the form

$$
p(M)=p\left(M_{2}\right) p\left(M_{1} \mid M_{2}\right) p\left(\left\{M_{k}, k \geq 3\right\} \mid M_{1}, M_{2}\right)
$$

where we in the following carefully explain the three factors in this expression in turn.

The $p\left(M_{2}\right)$ is essentially a prior distribution for the neighbourhood system $\Psi$, and for an arbitrary node $(i, j) \in \chi$ we assume apriori the various $(t, u) \in \chi \backslash\{(i, j)\}$ to be a neighbour of $(i, j)$ independently of each other, and with probabilities so that nodes close to $(i, j)$ have the highest probabilities of being a neighbour. More precisely, we define $p\left(M_{2}\right)$ to have the form

$$
p\left(M_{2}\right)=\prod_{\Lambda \in L: \tau(\Lambda)=2}\left[f_{\eta}(\Lambda)^{I\left(\Lambda \in M_{2}\right)}\left(1-f_{\eta}(\Lambda)\right)^{I\left(\Lambda \notin M_{2}\right)}\right]
$$

where $f_{\eta}(\Lambda) \in[0,1]$ is the probability for a specific node to be a neighbour, and $\eta$ is a parameter controlling this probability. In this paper we use

$$
f_{\eta}(\Lambda)=e^{-\eta d(\Lambda)}
$$




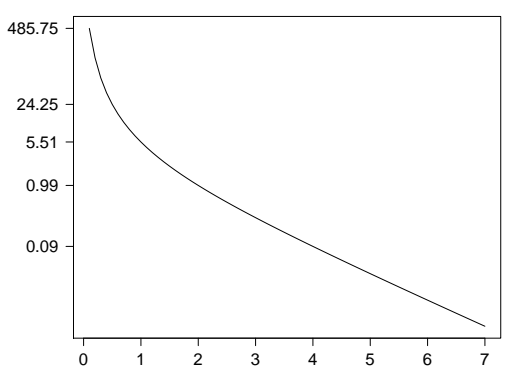

Figure 2: For a $100 \times 100$ lattice, the expected number of neighbours to a node, for given value of $\eta, 2 \cdot \mathrm{E}\left[\operatorname{card}\left(M_{2}\right) \mid \eta\right]$, as a function of $\eta$.

where $d(\Lambda)$ is the Euclidean distance between the two nodes in (an arbitrary) $\lambda \in \Lambda$ when $\tau(\Lambda)=2$. We consider $\eta$ as random and want to assign a prior distribution to it. For a given value of $\eta$ we can easily compute the expected number of neighbours of a node, see Figure 2 , As we can also understand from the definition of $p\left(M_{2}\right)$, the expected number of neighbours is very high for small values of $\eta$ and decreases rapidly as $\eta$ increases. Apriori we do not want high probability for the nodes to have a lot of neighbours, so the apriori density for $\eta$ should be small for values of $\eta$ close to zero, but otherwise we want the prior to be vague. We adopt a gamma prior for $\eta$ with mean 3 and standard deviation $\sqrt{3} \approx 1.73$. Close to zero this prior density is proportional with $\eta^{2}$, and our experience is that this is sufficiently small to avoid a very large number of neighbours when simulating from a posterior distribution.

When defining the factor $p\left(M_{1} \mid M_{2}\right)$ one should recall that one has only two possibilities for $M_{1}$, either $M_{1}=\emptyset$ or $M_{1}=\{\square\}$. Moreover, as we have assumed $M$ to be dense one must have $M_{1}=\{\square\}$ if $\operatorname{card}\left(M_{2}\right)>0$. Thereby we only need to specify prior probabilities $p\left(M_{1}=\emptyset \mid M_{2}=\emptyset\right)$ and $p\left(M_{1}=\{\square\} \mid M_{2}=\emptyset\right)$. We assume each of these probabilities to be a half, and get

$$
p\left(M_{1} \mid M_{2}\right)= \begin{cases}\frac{1}{2^{I\left(M_{2}=\emptyset\right)}} & \text { if } M_{1}=\{\square\} \text { or } M_{2}=\emptyset \\ 0 & \text { otherwise. }\end{cases}
$$

The conditional prior distribution $p\left(\left\{M_{k}, k \geq 3\right\} \mid M_{1}, M_{2}\right)$ is the probability for including clique types of orders larger than two, given $M_{1}$ and $M_{2}$ and under the restriction that $M$ should be dense. In particular we construct the rest of $M$ by adding sequentially the possible interactions of orders 3,4 and so forth, each with a probability $p_{\star}$. Given the second order interactions $M_{2}$ we first independently add the possible third order interactions with the 


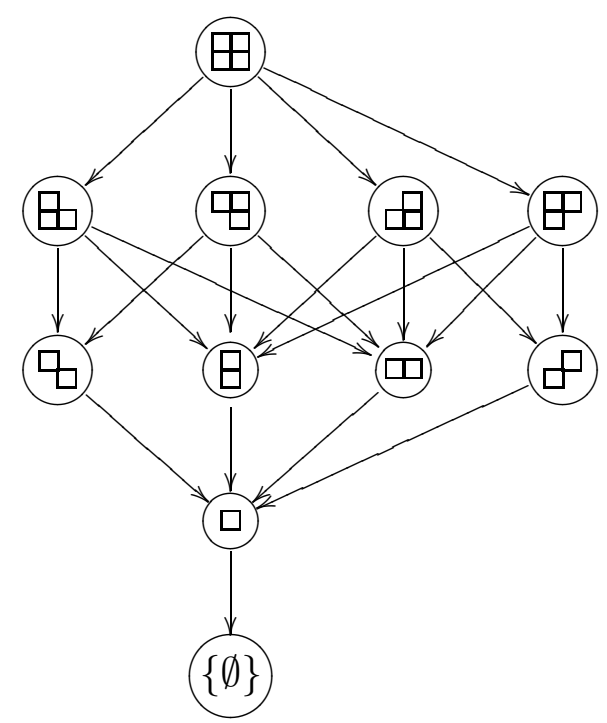

Figure 3: Maximal possible DAG when $M_{2}=\{$ ४, 曰,Ш,Ф\}.

probability $p_{\star}$. Then, given the set of third order interactions $M_{3}$ we independently add the possible forth order interactions with the same probability $p_{\star}$, and so forth. For instance, there are four possible third order interactions to add when the second order interactions are as given in Figure 1, namely $巴$ 由, 出, and 巴. If, in this example, all of the third order cliques are included it is also possible to include the forth order clique $\boxplus$, which gives us the maximal possible DAG for the $M_{2}$ in this example, see the illustration in Figure 3. For the $M$ illustrated in Figure 1, we see that only one of the third order cliques is included in $M$, and then clearly no forth order clique can be included without violating the restriction that $M$ should be dense. Defining $v_{k}\left(M_{k-1}\right)=\left\{\Lambda \in L ; \tau(\Lambda)=k\right.$ and $\Lambda^{\star} \prec \Lambda$ for all $\left.\Lambda^{\star} \in M_{k-1}\right\}$ to be the set of all clique types of order $k$ that is possible to include in $M$ given the clique types of order $k-1$ we get that

$$
p\left(\left\{M_{k}, k \geq 3\right\} \mid M_{1}, M_{2}\right)=\prod_{k \geq 3}\left\{\prod_{\Lambda \in v_{k}\left(M_{k-1}\right)}\left[p_{\star}^{I\left(\Lambda \in M_{k}\right)}\left(1-p_{\star}\right)^{I\left(\Lambda \notin M_{k}\right)}\right]\right\}
$$

when $M$ is dense. As a prior for $p_{\star}$ we assing a uniform distribution on the interval $(0,1)$.

Having defined the prior for $M$ as above, the next step in the prior specification is to define a prior that specify what sets of clique types that should have the same parameter value. We start by defining a partition of $M$, denoted $\mathscr{S}$, and use $S \in \mathscr{S}$ to denote a cell in 
that partition. We restrict all clique types in a cell $S$ to have the same parameter value, $\varphi_{S}$ say. By setting $z=\left\{\left(S, \varphi_{S}\right) ; S \in \mathscr{S}\right\}$ we thus have

$$
\phi^{\Lambda}=\sum_{\left(S, \varphi_{S}\right) \in z} \varphi_{S} I(\Lambda \in S)
$$

for $\Lambda \in M$, and we denote the vector of model parameters by $\varphi=\left(\varphi_{S} ; S \in \mathscr{S}\right)$. In the following we define a prior for $\left\{\phi^{\Lambda}, \Lambda \in M\right\}$ given $M$, by specifying a prior for $z$ given $M$. We specify this conditional prior for $z$ as

$$
p(z \mid M)=p(\mathscr{S} \mid M) p(\varphi \mid \mathscr{S}),
$$

where $p(\mathscr{S} \mid M)$ is a distribution over all partitions of $M$, and $p(\varphi \mid \mathscr{S})$ is a distribution for the parameters values given a partition $\mathscr{S}$.

For $p(\mathscr{S} \mid M)$ we first want to have a distribution that does not favour partitions with a high number of cells, as this would give a corresponding high number of model parameters. For instance, assigning a uniform distribution directly on all the possible partitions would make the marginal probability for approximately card $(M) / 2$ cells much higher than a state with only one cell. Second, we want the prior probability for any particular partition with $\operatorname{card}(\mathscr{S})=r$ cells to be smaller than any particular partition with $r+1$ cells. To see how one can construct a prior fulfilling these requirements one should note that it is possible to count the number of possible partitions of a set with card $(M)$ elements, noting that the definition of a partition require no cells to be empty. This is known as the Stirling numbers of the second kind (Ronald L. Graham 1988), and is for $\operatorname{card}(\mathscr{S})=r$ given by

$$
g(\operatorname{card}(M), r)=\frac{1}{r !} \sum_{k=0}^{r}\left(\begin{array}{l}
r \\
k
\end{array}\right)(-1)^{r-k} k^{\operatorname{card}(M)} .
$$

The function $g(\operatorname{card}(M), r)$ is strictly increasing as a function of $r$ from $r=1$ to $r=r_{\max }=$ $\arg \max _{r} g(\operatorname{card}(M), r) \approx \operatorname{card}(M) / 2$, and strictly decreasing from $r=r_{\max }$ to $r=\operatorname{card}(M)$. We define $p(\mathscr{S} \mid M)$ by a marginal distribution for the number of cells in the partition, $p(\operatorname{card}(\mathscr{S}) \mid M)$, and given the number of cells in $\mathscr{S}$ we assume a uniform distribution over all partitions with the specified number of cells. Thus, we have

$$
p(\mathscr{S} \mid M)=\frac{p(\operatorname{card}(\mathscr{S}) \mid M)}{g(\operatorname{card}(M), \operatorname{card}(\mathscr{S}))},
$$


and use $p(\operatorname{card}(\mathscr{S}) \mid M)$ to ensure that the prior fulfils the two properties discussed above. We define

$$
p(\operatorname{card}(\mathscr{S})=r \mid M) \propto \begin{cases}1 & \text { if } r<r_{\max }, \\ \frac{g(\operatorname{card}(M), r)}{g\left(\operatorname{card}(M), r_{\max }\right) 2^{r-r_{\max }}} & \text { otherwise }\end{cases}
$$

where the factor $2^{r}$ in the denominator grows faster than $g(\operatorname{card}(M), r)$ as a function of $r$. One should also note that the expression for $r \geq r_{\max }$ is chosen so that it is equal to 1 for $r=r_{\max }$. Finally we specify $p(\varphi \mid \mathscr{S})$ by assuming the components of $\varphi$ to be independent and normally distributed with zero mean and with some common variance $\sigma_{\varphi}^{2}$, but to make the model identifiable we add the restriction

$$
\sum_{\left(S, \varphi_{S}\right) \in z} \varphi_{S}=0
$$

To complete our fully Bayesian setup we assign a vague gamma distribution for $\sigma_{\varphi}^{2}$ with mean 10 and variance $10^{2}$.

\section{Posterior simulation}

In this section we briefly present how we simulate from the posterior distribution

$$
p(z, M, \theta \mid x) \propto p(x \mid z, M) p(z \mid M, \theta) p(M \mid \theta) p(\theta)
$$

where $p(x \mid z, M)$ is a likelihood MRF for an assumed observed scene $x, p(z \mid M, \theta)$ and $p(M \mid \theta)$ are the priors defined in the previous section, and $p(\theta)$ is the hyper-prior for the vector of prior parameters $\theta=\left(\eta, p_{\star}, \sigma_{\varphi}^{2}\right)$. When wanting to simulate from this distribution, two challenges arises. Firstly, as also briefly discussed in the introduction, this is a doubly intractable distribution (Murray et al. 2006). So in a Metropolis-Hastings algorithm the normalising constant for the MRF does not cancel in the expression for the acceptance probability. Secondly, we need to specify appropriate proposal distributions for $z, M$ and the prior parameter vector $\theta=\left(\eta, p_{\star}, \sigma_{\varphi}^{2}\right)$. In the following two sections we describe how we tackle these two problems. 


\subsection{Handling the normalising constant}

As in our case a MRF $x$ is usually defined conditioned on a parameter vector. The posterior distribution for such models are particularly computer intensive because the normalising constant becomes intractable as the dimension of $x$ increases, see (11). This is in the literature known as a doubly intractable distribution (Murray et al. 2006), and great efforts have been made in order to circumvent exact evaluations of the normalising constants. These techniques can roughly be divided into three approaches. The first is to introduce auxiliary variables in the Bayesian model in such a way that the resulting acceptance probabilities would be without any evaluation of the normalising constant. These approaches are discussed in Møller et al. (2006) and Murray et al. (2006). However to reach the true limiting distribution in such MCMC algorithms, we are required to simulate perfect samples (Propp and Wilson 1996) from the likelihood, which in turn can be very computationally demanding or even practically infeasible. If perfect simulation from the likelihood is infeasible Caimo and Friel (2011) and Everitt (2012) propose to replace the perfect sample with a sample generated by MCMC. Such an approach will not exactly target the correct posterior distribution, but the experience is that in many situations such a procedure provides a very good approximation to what we want. It should also be noted that the value of the parameter vector will typically restrict this approach as well, as the MCMC sampler for the auxiliary variable needs to converge for each sample.

A second approach for coping with the computationally intractable normalising constant is to replace it by an estimate obtained prior to posterior simulation. The estimate is usually obtained by an MCMC algorithm, which is also the approach taken in Geyer and Thompson (1992) and Higdon et al. (1997). This approach will however not give the correct limiting distribution, and for the approach to be practical it usually requires the dimension of the parameter vector to be low. The third option for handling the intractable normalising constant is to replace the likelihood by an approximation, and thereby instead simulate from an approximation to the posterior distribution. Several approximation schemes have been developed, including approximation by pseudo-likelihood (Heikkinen and Högmander 1994), reduced dependency approximation (Friel et al. 2009; McGrory et al. 2012), and approxima- 
tion by pseudo-Boolean functions (Austad 2011; Tielmeland and Austad 2012). As with the second approach we will not get the true limiting distribution using approximations, however there are typically no computational restrictions on the dimension and value of the parameter vector, although the quality of the approximation may depend on these quantities.

In our situation we have an MRF with a parameter vector of possibly a somewhat high dimension, and in fact we have also put a prior on its dimension, so the second approach is not a viable alternative. We have tried both the first approach, with an MCMC sample to replace the perfect sample, and the third approach using pseudo-Boolean approximations. For our examples we found the first approach to be computationally cheaper and sufficiently accurate, so in our simulation examples we present results when adopting this approach.

\subsection{Proposal distributions}

In this section we only give a brief description of the proposal distributions we have used when simulating from the posterior distribution. A more detailed description is given in the supplementary material to this paper, see Section S.1. In our algorithm we combine six proposal distributions making changes in $z$ or $M$, and three proposal distributions modifying the hyper-parameter vector $\theta$. Each of the proposals for $z$ or $M$ is made according to a proposal probability vector $p_{o}=\left(p_{o}^{1}, p_{o}^{2}, p_{o}^{3}, p_{o}^{4}, p_{o}^{5}, p_{o}^{6}\right)$, where each of these probabilities correspond to the order in which we discuss the proposals here. Our first proposal is a random walk proposal for the $\varphi$ parameters, adjusting for the sum-to-zero constraint. Our second proposal is to take one clique type in a given cell and propose to change its cell membership. In the third proposal we propose to decrease or increase the number of cells by one, changing the dimension of the parameter vector $\varphi$ correspondingly. As a forth proposal we propose to replace a clique type that is on by a different clique type, copying the parameter value and cell membership from the old clique type to the new one. In the fifth proposal we propose to turn on a new clique type or turn off an already existing clique type. When proposing to turn on a new clique type we propose to insert the new clique type into an already existing cell, and when proposing to turn off an existing clique type we choose to delete a clique type from a cell with size larger than one, preserving the dimension of the parameter vector in both cases. In the sixth and last of the proposals for $z$ and $M$, we 
propose to turn on a clique type and assigning it to it a new cell, or to turn off a clique type that is the only clique type in its cell. This proposal will also result in a dimension change in the parameter vector $\varphi$. Note that we allow some of these proposals to lead to states of zero prior probability. However for such proposals we do not need to evaluate the likelihood, so such proposals are very cheap to evaluate. The first three of the proposals described above make changes to already existing parameter values or to the cells of the partition. The last three proposals also makes changes to $M$, i.e. to what clique types that are on or off. In the supplementary materials we give the proposals we use when updating the elements of $\theta$. These proposals are cheap to evaluate and we do one of each of these for each time we perform one of the six proposals described above.

\section{Simulation examples}

In this section we investigate our prior and proposal distributions on three examples. Firstly we consider a simulation example where data is generated from an Ising model, secondly we generate data from a model with $2 \times 2$ cliques, and thirdly we look at a real data example consisting of presence and absence of red deer. In all our simulations we evaluate the acceptance probabilities by using the exchange algorithm of Murrav et al. (2006) with a Gibbs sampler to obtain the auxiliary variables (Caimo and Friel 2011). The length of the burn in period of the Gibbs sampler are in all simulations set to 200 iterations, although experiments suggest that only about half of is sufficient in all our examples. We set $p_{o}=$ $(0.1,0.15,0.15,0.15 .0 .20,0.25)$ for the two first examples and a slightly different $p_{o}$ for the last example, see Section 7.3. For the tuning parameters described in the supplementary materials, see Section S.1, we set $\sigma_{w}=0.2, \sigma_{g}=\sigma_{c}=0.3, \sigma_{\eta}=2.0, \sigma_{\sigma_{\varphi}}=0.7$ and $\rho=0.1$, based on some preliminary runs.

\subsection{The Ising model}

In this section we used a perfect sampler (Propp and Wilson 1996) to generate one realisation from an Ising model on a $100 \times 100$ lattice with torus boundary conditions. The Ising model 


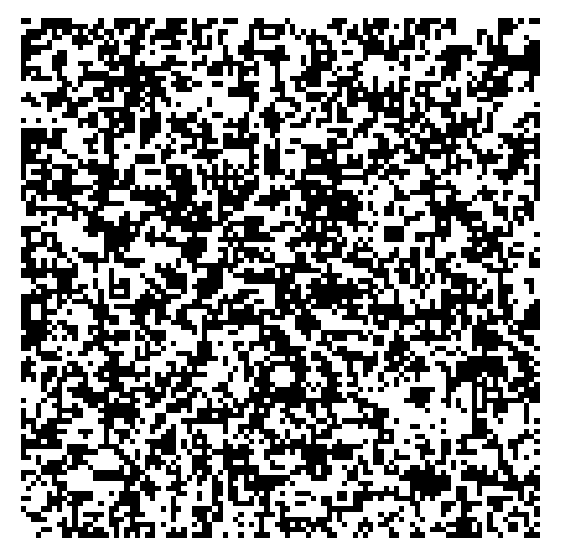

Figure 4: Ising example: Data simulated from the Ising model in (13).

used is given by

$$
p(x)=\exp \left(0.4 \sum_{(i, j) \sim(k, l)} I\left(x_{i, j}=x_{k, l}\right)\right),
$$

where the sum is over all pairs of first order neighbour nodes, taking the torus boundary assumption into account. The realisation used is shown in Figure 4. This Ising model corresponds to the set of clique types $M=\{\{\emptyset\}, \square, \square, \boxminus\}$ with the partition either being $\mathscr{S}=$ $\{\{\{\emptyset\}\},\{\square\},\{\square, \boxminus\}\}$ or $\mathscr{S}=\{\{\{\emptyset\}\},\{\square\},\{\square\},\{\boxminus\}\}$. The true parameter values for these two model formulations are $\varphi_{\{\{\emptyset\}\}}=1.3333, \varphi_{\{\square\}}=-0.2666 \varphi_{\{\square \square}=-1.0666$, and $\varphi_{\{\{\emptyset\}\}}=1.6$, $\varphi_{\{\square\}}=0, \varphi_{\{\square\}}=-0.8, \varphi_{\{\boxminus\}}=-0.8$ respectively. To simulate from the posterior in (12) we ran our sampler for $10^{6}$ iterations, and based on convergence plots we could see that the MCMC sampler had converged after about $10^{4}$ iterations. The acceptance rates for each of the proposals changing $z$ and $M$ were for this example $0.102,10^{-5}, 0.002,10^{-4}, 10^{-4}$ and 0.001, given in the same order as in $p_{o}$. The reason for the very low figures for some of these acceptance rates is in fact not slow mixing, but just that the posterior distribution has essentially all its mass in one state for $M$ and $\mathscr{S}$. The acceptance rates for the three updates of $\eta, p_{\star}$ and $\sigma_{\varphi}^{2}$ were $0.278,0.499$ and 0.462 respectively.

Discarding the burn-in period of $10^{4}$ iteration, we now investigate the posterior distribution of $M, \mathscr{S}$, the parameter values $\varphi$, and the hyper-parameters $\eta, p_{\star}$ and $\sigma_{\varphi}^{2}$. For $M$ the estimated posterior probability of the correct state becomes 0.999, so the sampler just very briefly visit other states. If we consider only the realisations that consist of the correct state of $M$, there are only two partitions that are simulated, namely the two partitions given 
above. The three and four cell partitions have estimated posterior probabilities equal to 0.991 and 0.009 respectively, so the model with less parameters is preferred.

To evaluate the posterior distribution for the parameters is complicated when simulating from a distribution where not only the parameter values, but also the dimension and interpretation of the parameters vary, see for example the discussion in Celeux et al. (2000). In our model the interpretation of both $\varphi$ and $\left\{\phi^{\Lambda}, \Lambda \in M\right\}$ vary with $M$ and $\mathscr{S}$, so to study the posterior distribution of the elements of these vectors across different states for $M$ and $\mathscr{S}$ is of no value. Recalling that $\phi^{\Lambda}$ is well defined also when $\Lambda$ is off, $\Lambda \notin M$, we instead focus on $\phi^{\Lambda}-\phi^{\{\emptyset\}}, \Lambda \in L \backslash\{\{\emptyset\}\}$ which has the same interpretation for all $M$ and $\mathscr{S}$. From (3) we see that $\phi^{\Lambda}-\phi^{\{\emptyset\}}=U\left(\mathbf{1}^{\lambda}\right)-U\left(\mathbf{1}^{\emptyset}\right)$ for any $\lambda \in \Lambda$. We in particular choose to focus

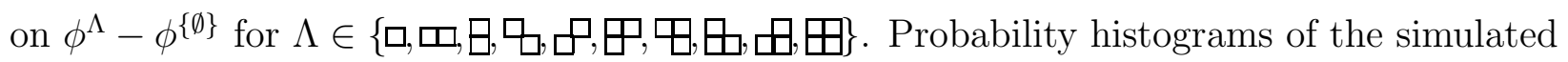
values for these ten statistics are shown in Figure 5. The corresponding values for the Ising model in (13) that we used to generate the data set we are analysing now are shown as black boxes on the $x$-axis, and we see in fact that the correct $\phi^{\Lambda}-\phi^{\{\emptyset\}}$ values are well recovered.

For the prior parameters $\eta, p_{\star}$, and $\sigma_{\varphi}^{2}$ we get the results shown in Figure 6. The median of the posterior samples of $\eta$ is 1.62 with $95 \%$ credible interval $(0.931,3.331)$. The posterior distribution for $p_{\star}$ is essentially uniform, which is reasonable since there is no possibility for higher order interactions in most of the posterior realisations and thereby this parameter is in most of the iterations just simulated from the prior. The median of $\sigma_{\varphi}^{2}$ is 3.857 in this example.

\section{$7.2 \quad$ A $2 \times 2$ maximal clique model}

In the next simulation experiment we generated a data set from an MRF with $2 \times 2$ maximal cliques and $M$ as shown in Figure 3 and $\mathscr{S}$ and parameter values as shown in Table 1 . Just as for the Ising model discussed above there are of course several possibilities for $\mathscr{S}$ specifying the model we are using, so in Table 1 we give $\mathscr{S}$ with the lowest number of elements. We also give the corresponding values of $\phi^{\Lambda}-\phi^{\{\emptyset\}}$ for $\Lambda \in L \backslash\{\{\emptyset\}\}$ as the interpretation of these perhaps is easier, and because we want to compare these values with corresponding values for the posterior samples. We were not able to obtain a perfect sample by coupling from the past for this model, so we generated instead a realisation by a Gibbs sampling algorithm, 


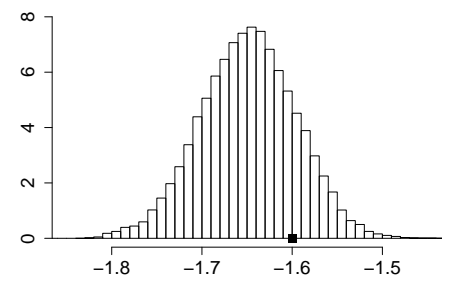

(a) $\phi \square-\phi^{\emptyset}$

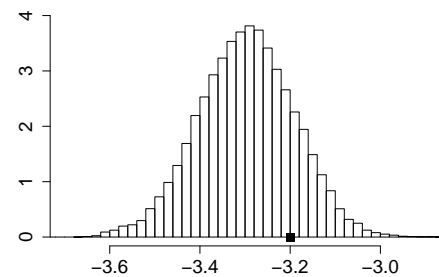

(d) $\phi \square-\phi^{\emptyset}$



(g) $\phi$ 廿 $\phi^{\emptyset}$

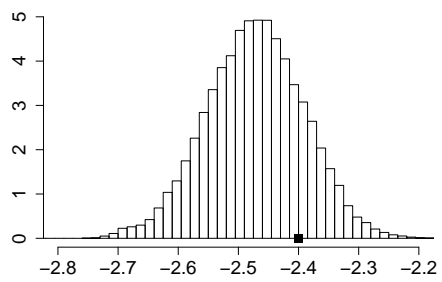

(b) $\phi \varpi-\phi^{\emptyset}$

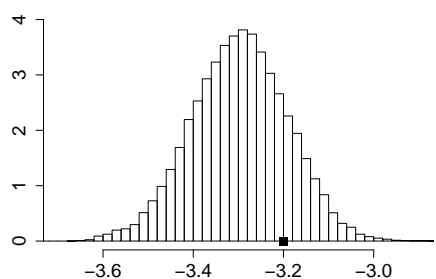

(e) $\phi \square-\phi^{\emptyset}$

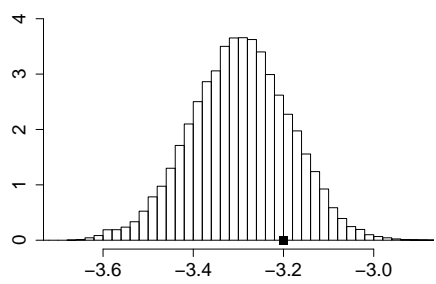

(h) $\phi \boxminus-\phi^{\emptyset}$



(j) $\phi \boxplus-\phi^{\emptyset}$

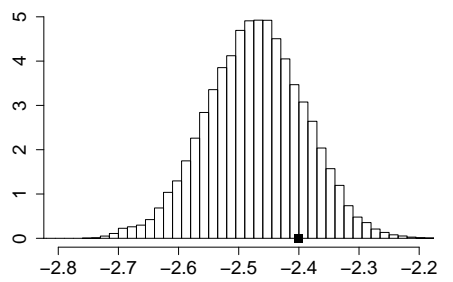

(c) $\phi$ 日 $-\phi^{\emptyset}$

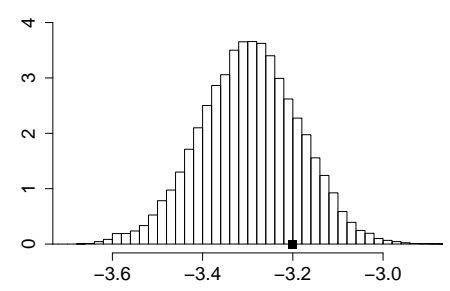

(f) $\phi \boxminus-\phi^{\emptyset}$

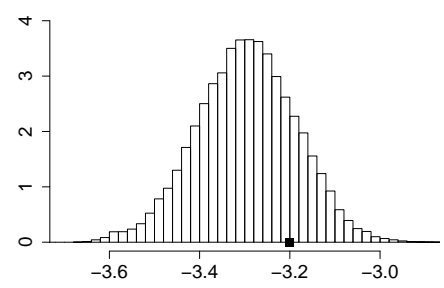

(i) $\phi \boxplus-\phi^{\emptyset}$

Figure 5: Ising example: Probability histograms for the posterior simulated values for $\phi^{\Lambda}-\phi^{\{\emptyset\}}$ for ten values of $\Lambda$. True values are shown with a black box.

and the generated data is shown in Figure 7. This data set contains both small areas with clustering of black or white nodes and small areas with a checkerboard pattern.

Also for this example we simulate from the posterior distribution by running our sampler for $10^{6}$ iterations. Based on convergence plots we concluded that the sampler had converged after about $10^{5}$ iterations. The acceptance rates for the six proposals for $z$ and $M$ were $0.062,0.022,0.023,10^{-4}, 10^{-4}$ and $10^{-4}$, again given in the same order as in 


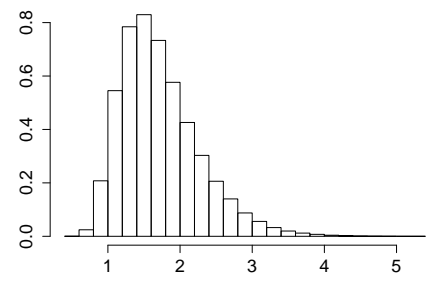

(a) $\eta(0.931,3.331)$

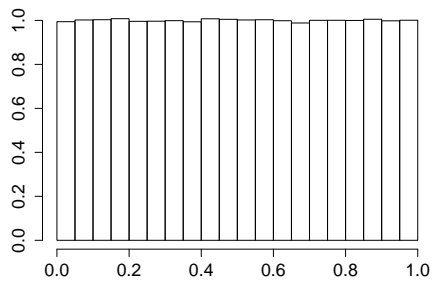

(b) $p_{\star}(0.025,0.975)$

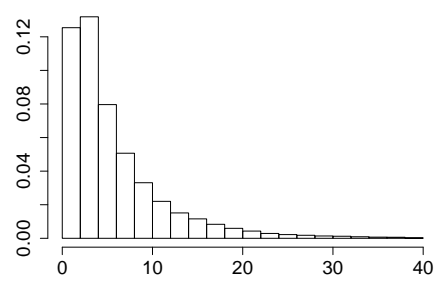

(c) $\sigma_{\varphi}^{2}(0.665,21.694)$

Figure 6: Ising example: Probability histograms for the hyper-parameters $\eta, p_{\star}$ and $\sigma_{\varphi}^{2}$, with $95 \%$ credible intervals.

\begin{tabular}{|c|c|}
\hline$\Lambda \in L \backslash\{\{\emptyset\}\}$ & $\phi^{\Lambda}-\phi^{\{\emptyset\}}$ \\
\hline$\square$ & -0.10 \\
\hline ㅁ. 日 & -0.75 \\
\hline ६, 口 & 0.20 \\
\hline 由,由, 由, 由 & -1.05 \\
\hline 田 & -1.10 \\
\hline
\end{tabular}

\begin{tabular}{|c|c|}
\hline$S \in \mathscr{S}$ & $\varphi_{S}$ \\
\hline$\{\{\emptyset\}\}$ & 0.4667 \\
\hline$\{\square\}$ & 0.3667 \\
\hline$\{\square, 日\}$ & -0.2833 \\
\hline$\{\square, \square\}$ & 0.6667 \\
\hline$\{巴$, 由, 由, 円\} & -0.5833 \\
\hline$\{\boxplus\}$ & -0.6333 \\
\hline
\end{tabular}

Table 1: MRF example with $2 \times 2$ cliques: True parameter setting with associated (minimal) partition.

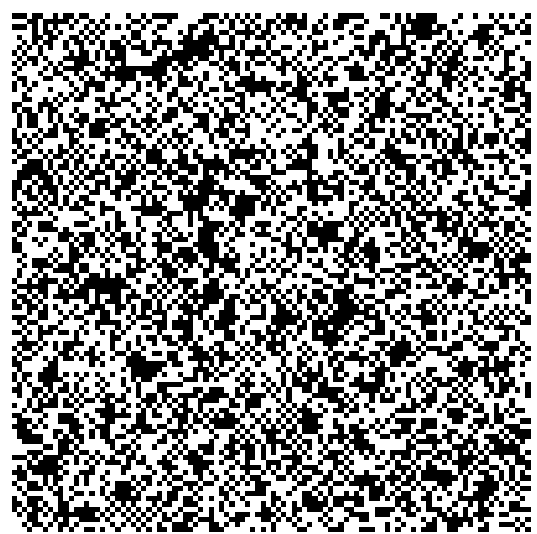

Figure 7: MRF example with $2 \times 2$ cliques: Data simulated from the $2 \times 2$ model with parameters given in Table 1 .

$p_{o}$, while the acceptance rates for $\eta, p_{\star}$ and $\sigma_{\varphi}^{2}$ was $0.203,0.333$ and 0.347 , respectively. As in the previous example we investigate the posterior distribution of $M, \mathscr{S}, \phi^{\Lambda}-\phi^{\{\emptyset\}}$

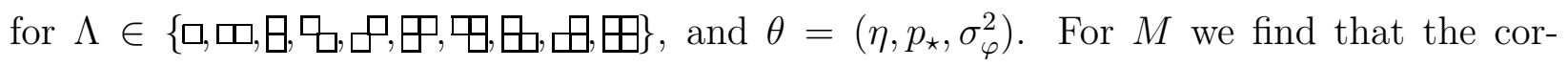
rect model are simulated with probability 0.99. The estimated most probable partition, however, differ slightly from the true model. The aposteriori most probable partition is 


\begin{tabular}{|c|c|c|}
\hline$\hat{P}(\mathscr{S} \mid M)$ & $\mathscr{S}$ & $\operatorname{card}(\mathscr{S})$ \\
\hline 0.223 & 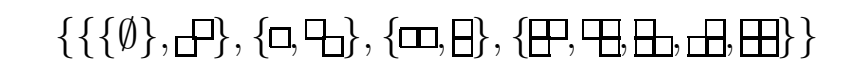 & 4 \\
\hline 0.091 & 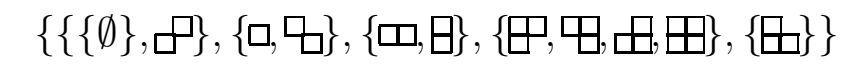 & 5 \\
\hline 0.089 & 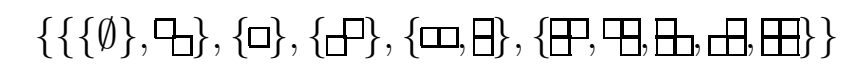 & 5 \\
\hline 0.049 & $\{\{\{\emptyset\}$, 口 $\},\{\square\},\{\square\},\{\square, 日\},\{\boxplus, \boxplus, \boxplus, \boxplus, \boxplus\}\}$ & 5 \\
\hline 0.046 & 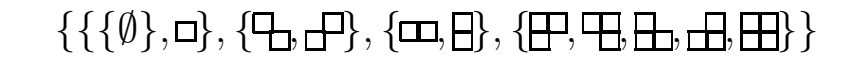 & 4 \\
\hline 0.023 & 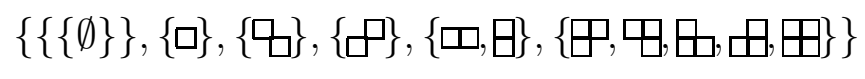 & 6 \\
\hline 0.022 & $\{\{\{\emptyset\}, \square\},\{\square$, ४ $\},\{$ 口, 日 $\},\{\boxplus$, 田, 田 $\},\{\boxplus\}\}$ & 5 \\
\hline 0.021 & 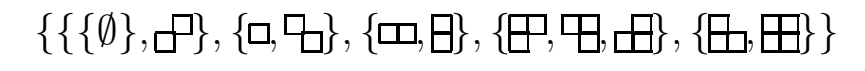 & 5 \\
\hline
\end{tabular}

Table 2: MRF example with $2 \times 2$ cliques: The aposteriori ten most probable partitions.

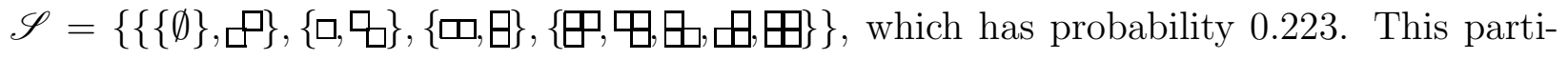
tion differs from the true partition in that the cell $\{$ ६, $\square\}$ is removed and ५ and $\square$ are instead inserted into the cells $\{\{\emptyset\}\}$ and $\{\square\}$, respectively, and in that the two cells $\{\boxplus\}$ and $\{$ 田,由,由\} in the true partition are merged into one cell. In total eight partitions have probabilities larger than 0.02 , and these are shown in Table 2. Additional ten partitions have posterior probabilities larger that 0.01 (not shown). After the burn-in, the MCMC run visited a total 370 partitions for the most probable state of $M$, and the partition used to generate the data set is the aposteriori 75th most probable partition, with an estimated posterior probability of approximately $10^{-4}$. Looking at the most probable partitions given in Table 2 we notice that except one, all of these partitions have one or two cells less than that of the true partition. Because of the relatively week interactions in our data set the posterior prefers partitions corresponding to a lower number of parameters. However, as shown in Figure 8 , the true $\phi^{\Lambda}-\phi^{\{\emptyset\}}$ values are still not completely missed by the posterior.

The multi-modality of the probability histograms for $\phi^{\Lambda}-\phi^{\{\emptyset\}}$ can be understood. For example the spike at zero in Figure $8(\mathrm{a})$ is due to $\square$ and $\{\emptyset\}$ being assigned to the same cell. Similarly the spikes at zero in the distributions for ६ and $\square$ are the result of these clique types being assigned in a cell with $\{\emptyset\}$. Because of the weak interactions in the data, the tendency to have too few cells in the partition, compared to the true solution, makes the posterior samples often miss the true value of $\phi^{\Lambda}-\phi^{\{\emptyset\}}$. However one can argue that this is 


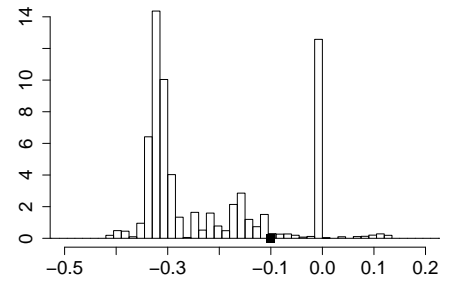

(a) $\phi \square-\phi^{\emptyset}$

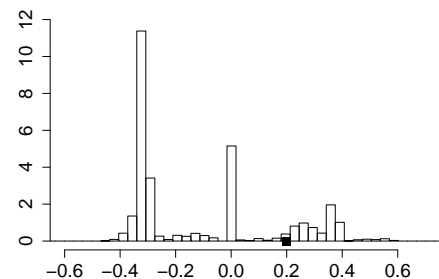

(d) $\phi \square-\phi^{\emptyset}$

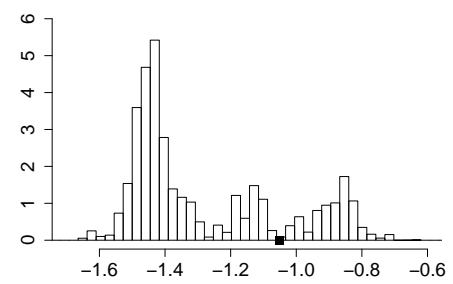

(g) $\phi$ 母- $\phi^{\emptyset}$

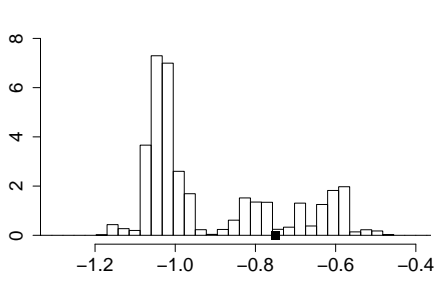

(b) $\phi \varpi-\phi^{\emptyset}$

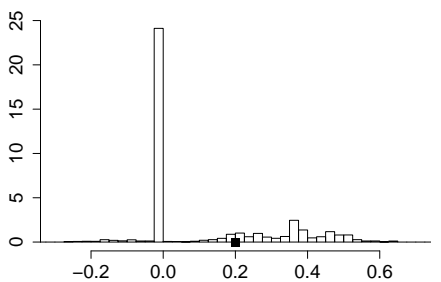

(e) $\phi \square-\phi^{\emptyset}$

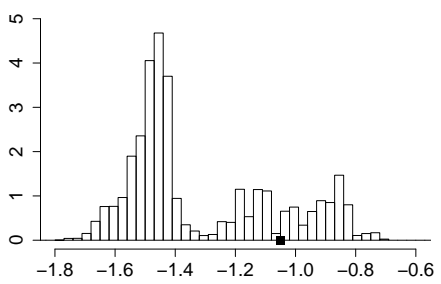

(h) $\phi \boxminus-\phi^{\emptyset}$

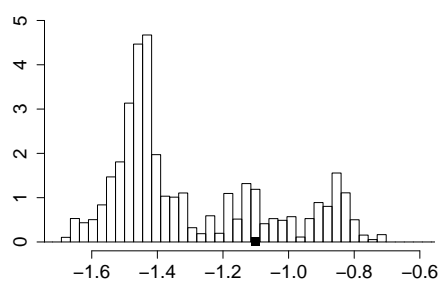

(j) $\phi$ 田 $-\phi^{\emptyset}$

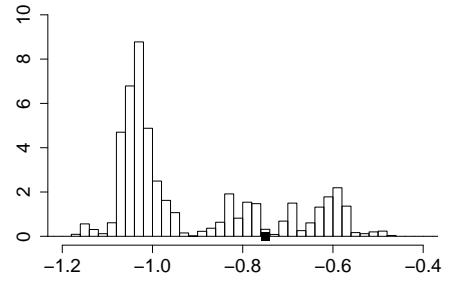

(c) $\phi-\phi^{\emptyset}$

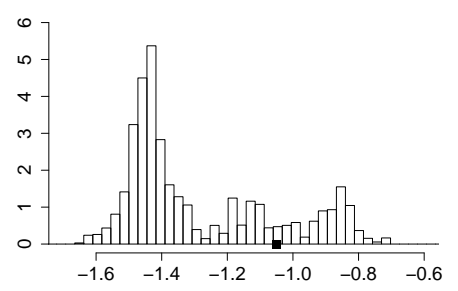

(f) $\phi \boxplus-\phi^{\emptyset}$

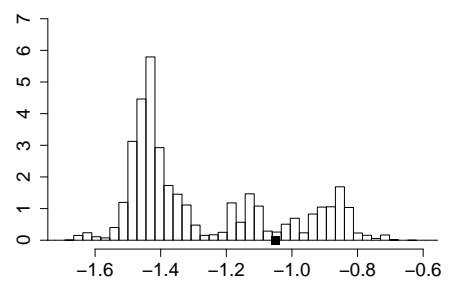

(i) $\phi \boxminus-\phi^{\emptyset}$

Figure 8: MRF example with $2 \times 2$ cliques: Probability histograms for the posterior simulated values for $\phi^{\Lambda}-\phi^{\{\emptyset\}}$ for ten values of $\Lambda$. True values are shown with a black box.

done without a dramatic decrease in the likelihood. This last claim can be investigated for instance by simulating from the likelihood using some of the model and associated parameters produced by the posterior in the MCMC run. Three such simulations from the likelihood for three different parameter settings are shown in Figure 9, where the last of these realisations is from a parameter setting from the most probable partition given in Table 2. As we can see the same three phase patterns as in the original data is present in all of these simulations. 

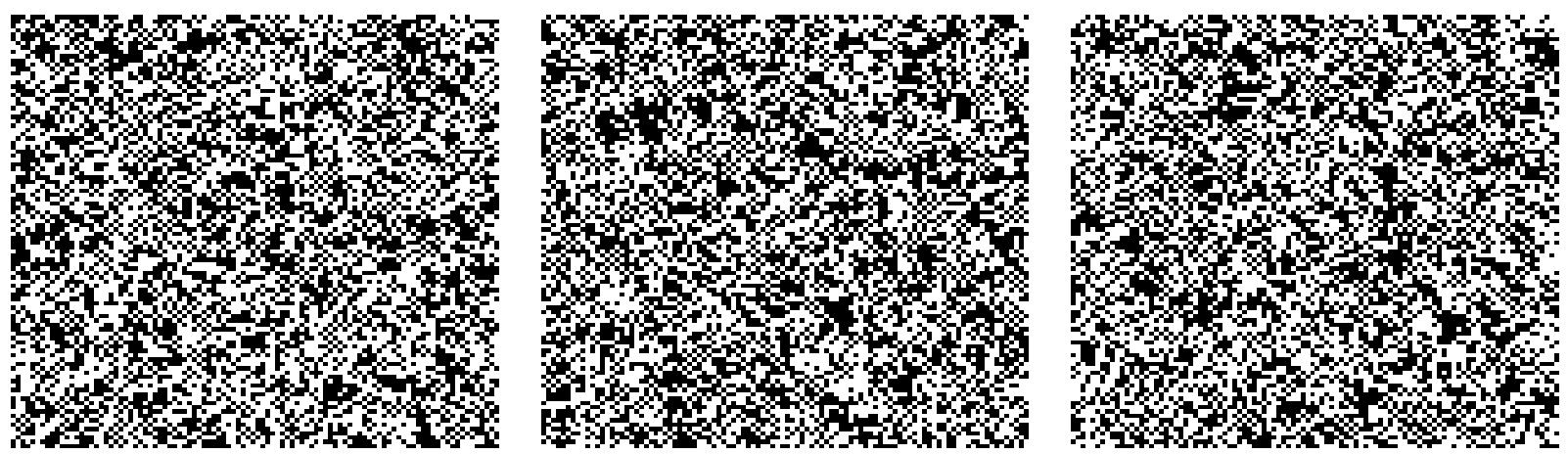

Figure 9: MRF example with $2 \times 2$ cliques: Simulations for three parameter realisation.

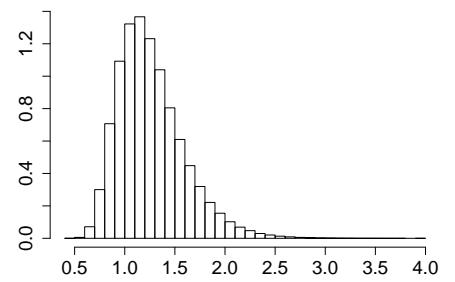

(a) $\eta(0.767,2.029)$

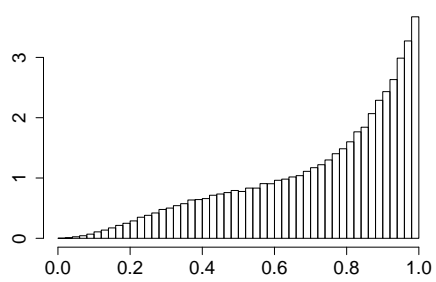

(b) $p_{\star}(0.216,0.994)$

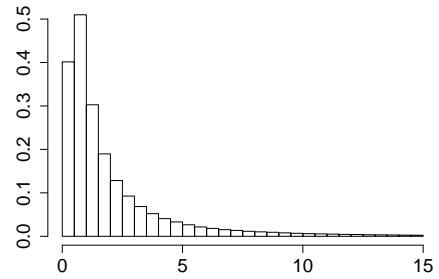

(c) $\sigma_{\varphi}^{2}(0.210,10.437)$

Figure 10: MRF example with $2 \times 2$ cliques: Prior-parameters with $95 \%$ credible interval.

In addition the strength of the interaction between the nodes seems similar as well.

For the prior parameters $\eta, p_{\star}$, and $\sigma_{\varphi}^{2}$ we get the posterior probability histograms shown in Figure 10. The posterior median for $\eta$ is 1.207 in this example. The estimated posterior median for $p_{\star}$ is 0.794 , which is reasonable given that this represents the probability for turning on clique types $\Lambda$ where $\tau(\Lambda)>2$. Thus, the shape of the posterior distribution have higher probability for values for $p_{\star}$ that favours higher order clique types to be on. The estimated median for $\sigma_{\varphi}^{2}$ is equal to 1.133 in this example.

\subsection{Red deer example}

In this section we consider a data set consisting of the classes absence(0) and presence(1) of reed deer in the Grampians Region of north-east Scotland, see Figure[11. A full description of the data set is given in Augustin et al. (1996) and Buckland and Elston (1993), and the data set has previously been analysed in Tjelmeland and Austad (2012) and Arnesen and Tjelmeland (2015). Our focus is to analyse the spatial interaction in the observed values by adopting the prior specified in Section 5. 

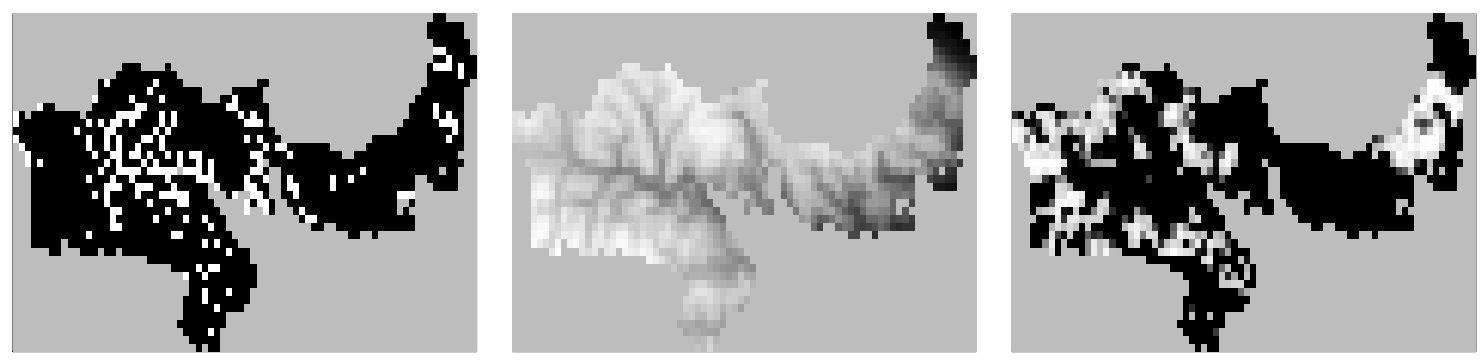

Figure 11: Red deer example: The presence/absence of red deer (left), altitude (middle), and mires (right) in the Grampians Region of north-east Scotland.

The observed values do not lie on a rectangular lattice, and to cope with this we include the observed $x$ into a larger lattice $x_{l}=\left(x, x_{b}\right)$ such that $x_{l}$ is a rectangular lattice where every element of $x$ is separated from the boundary by at least one node in $x_{b}$. For this extended lattice we then assume torus boundary conditions. In simulation experiments not discussed here we have also tried to increase the size of the extended lattice, but we then obtained essentially the same results as discussed below.

In addition to the binary values shown in Figure 11, we also have four covariates available in each of the nodes, namely altitude, mires, and north and east coordinates. We denote the covariate $k$ at a location $(i, j)$ by $y_{i, j, k}, j=1,2,3,4$, and model them into the likelihood by

$$
p\left(x_{l} \mid z, M, \kappa, y\right)=Z \exp \left(U\left(x_{l} \mid z, M\right)+\sum_{(i, j) \in \chi} x_{i, j} \sum_{k=1}^{4} \kappa_{k} y_{i, j, k}\right),
$$

where $U\left(x_{l} \mid z, M\right)$ is an energy function modelling spatial interaction as discussed in Sections 4, and $\kappa=\left(\kappa_{1}, \ldots, \kappa_{4}\right)$ are parameters associated to each of the four covariates. On $z$ and $M$, and the associated hyper-parameter $\theta$, we assign a prior as discussed in Section 5 and, independent of $z, M$ and $\theta$, we adopt independent zero mean Gaussian priors with standard deviation equal to 10 on $\kappa_{1}, \ldots, \kappa_{4}$.

The resulting posterior distribution of interest is

$$
p\left(z, M, \theta, \kappa, x_{b} \mid x\right) \propto p\left(x_{l} \mid z, M, \kappa, y\right) p(z \mid M, \theta) p(M \mid \theta) p(\theta) p(\kappa) .
$$

When simulating from this distribution we let each iteration consist of one of the six updates for $z$ and $M$ discussed in 6.2 or one update of a randomly chosen element in $\kappa$, a Gibbs scan for the elements in $x_{b}$, and updates of the elements in $\theta$ as discussed in Section 6.2. Therefore 
we increase the dimension of $p_{o}$, in this example, to include updates of the elements in $\kappa$ as well. We set $p_{o}=(0.1,0.1,0.15,0.15,0.20,0.25,0.05)$ where the first six entries are the probabilities as defined before and the last entry is the probability to propose a change for one of the elements in $\kappa$. To generate a potential new value for a chosen element in $\kappa$ we add to the current value a zero mean normally distributed random variable with standard deviation 0.2. For this example we ran the sampler for $10^{8}$ iterations, and investigation of convergence plots suggested convergence after about $10^{5}$ iterations. The acceptance rates for the proposals of $z, M$ and $\kappa$ were $0.207,0.026,0.043,0.005,10^{-3}, 0.002$ and 0.268 , where these rates are given in the same order as in $p_{o}$, and in particular the last acceptance rate is for the proposal of the covariates $\kappa$. The acceptance rates for $\eta, p_{\star}$ and $\sigma_{\varphi}^{2}$ was $0.212,0.349$ and 0.469 respectively in this example.

Again we investigate the posterior distribution of $z$ and $M$ after convergence. In total the chain visited more than 10000 different states of $M$ after convergence, and there are nine models with estimated posterior probability larger that 0.02 , all given in Table 3 . Together with posterior probabilities for $M$ and the most probable partition under $M$, the associated neighbourhoods are also shown in this table. Note that all the neighbourhoods in this table suggest some anisotropy from up-left to down-right. In addition to the nine states in Table 3. 12 additional states for $M$ have probabilities larger that 0.01 .

The estimated distribution for $\phi^{\Lambda}-\phi^{\{\emptyset\}}$ for the same $\Lambda \mathrm{s}$ as considered in the simulation examples, and in addition for $\Lambda \in\left\{\square_{\square},{ }_{\square}\right\}$, are shown in Figure 12, Several of these histograms are bimodal. In Section S.2 in the supplementary material we investigate this bimodality more in detail. In particular we show that the bimodality can be explained by splitting the posterior realisations into two groups. If we consider only realisations under the most probable posterior set of clique types $M=\{\{\emptyset\}, \square, \square, \Theta$, ४, 廿,由 $\}$, see Table 3, we get unimodal distributions for the $\phi^{\Lambda}$ values where its mode belongs to the first mode in Figures 12(a), (e), (k) and (l), and to the second mode in the rest of these plots. The second group of realisations consists of the remaining realisations, and in fact the distribution of $\phi^{\Lambda}$ for this group is again bimodal for some $\phi^{\Lambda}$. This bimodality can in turn by explained by looking at the realisations separately for $\phi^{\Lambda}$ when $\Lambda$ is either on or off, again see Section $\mathrm{S} .2$ in the supplementary materials for details. It is also worth mentioning that most of the 


\begin{tabular}{|c|c|c|c|}
\hline$\hat{P}(M)$ & $M$ with partition $\mathscr{S}_{\max }=\max _{\mathscr{S}} \hat{P}(\mathscr{S} \mid M)$ & $\hat{P}\left(\mathscr{S}_{\max } \mid M\right)$ & Neighbourhood \\
\hline 0.284 & $\{\{\{\emptyset\}\},\{\square\},\{$ 日,口, ६ $\},\{$ 吕, 由 $\}\}$ & 0.870 & 兩 \\
\hline 0.061 & $\left\{\{\{\emptyset\}\},\{\square\},\left\{\square, \Theta, \square_{\square},{ }_{\square}^{\square}\right\}\right\}$ & 0.855 & \\
\hline 0.061 & $\left\{\{\{\emptyset\}\},\{\square\},\left\{\theta^{\prime} \square_{\square},{ }_{\square}^{\square}\right\}\right\}$ & 0.953 & \\
\hline 0.041 & $\left\{\{\{\emptyset\}\},\{\square\},\left\{\square^{\prime}, \square_{\square}{ }_{\square}^{\square}\right\}\right\}$ & 0.923 & \\
\hline 0.030 & $\left\{\{\{\emptyset\}\},\{\square\},\left\{\square, \square,{ }_{\square}\right\}\right\}$ & 0.799 & 为 \\
\hline 0.026 & $\{\{\{\emptyset\}\},\{\square\},\{\square, \boxminus, \square \square\}\}$ & 0.829 & 戌 \\
\hline 0.026 & $\left\{\{\{\emptyset\}\},\{\square\},\left\{\square, \Theta,{ }^{\square}\right\}\right\}$ & 0.797 & \\
\hline 0.025 & $\{\{\{\emptyset\}\},\{\square\},\{\square, \square \square, \square\}\}$ & 0.901 & $\frac{\mathrm{x}}{\mathrm{x} \cdot \mathrm{y}}$ \\
\hline 0.024 & $\{\{\{\emptyset\}\},\{\square\},\{$ 曰,, 口 $\square\}\}$ & 0.758 & 爾 \\
\hline
\end{tabular}

Table 3: Red deer example: The realisations of $M$ with posterior probability higher than 0.02. First column: Estimated posterior probability of a specific $M$. Second column: Partition $\mathscr{S}_{\max }$ with the highest posterior probability for the given $M$. Third column: Estimated posterior probability for $\mathscr{S}_{\max }$ given $M$. Last column: Neighbours (shown with $\times$ ) for a node (shown with ·) resulting from $M$.

most probable set of clique types with posterior probability smaller than 0.02 , i.e. the most probable realisations of $M$ not shown i Table 3, are states containing different combinations of the second order clique types $\square$, 日, 只, $\square_{\square}$ and ${ }_{\square}$, which are the ones also used for the most probable set of clique types given in Table 3. In fact, the posterior probability that $M$ is a subset of $\left\{\{\emptyset\}, \square, \square, \boxminus, \square, \square_{\square}, \square\right\}$ but not equal to $\{\{\emptyset\}\}$ or $\{\{\emptyset\}, \square\}$ is as high as 0.307 . Also, the posterior probability that at least one clique type from the set $\left\{\square, \Theta, \nvdash^{\prime}, \square_{\square},{ }_{\square}\right\}$ is included in $M$ is very close to unity. In other words, these second order clique types seem to be the building blocks of the dependency structure in this data set. 


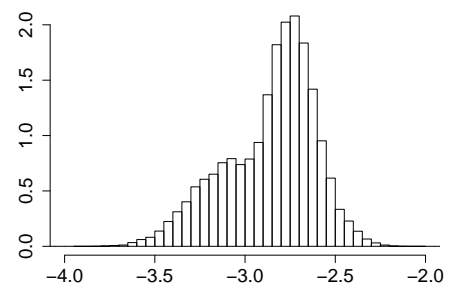

(a) $\phi \square-\phi^{\{\emptyset\}}$

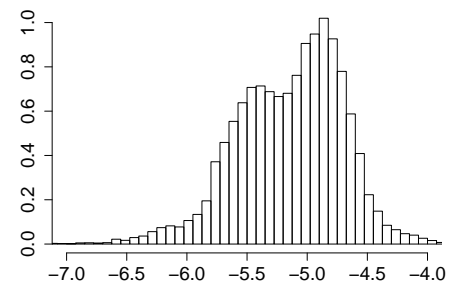

(d) $\phi \square-\phi^{\{\emptyset\}}$

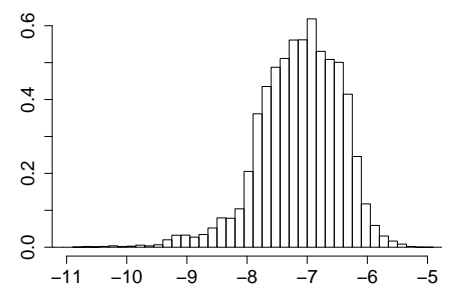

(g) $\phi$ 廿 $\phi^{\{\emptyset\}}$

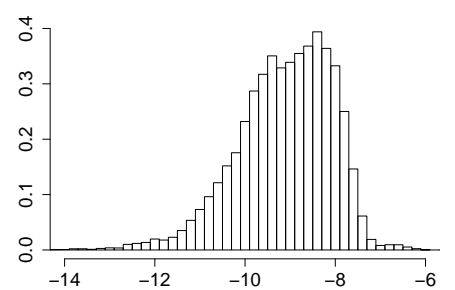

(j) $\phi \boxplus-\phi^{\{\emptyset\}}$

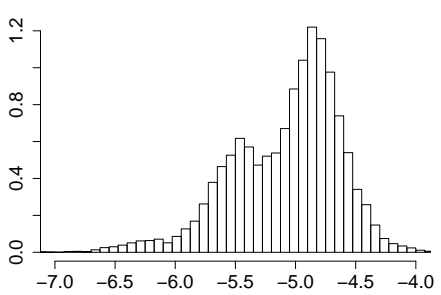

(b) $\phi \boxminus-\phi^{\{\emptyset\}}$



(e) $\phi \square-\phi^{\{\emptyset\}}$

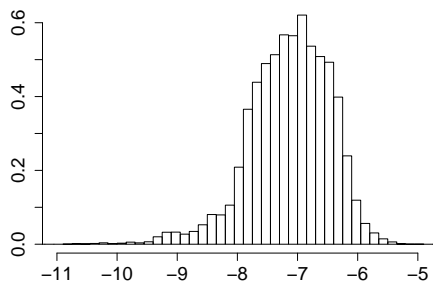

(h) $\phi$ В $-\phi^{\{\emptyset\}}$

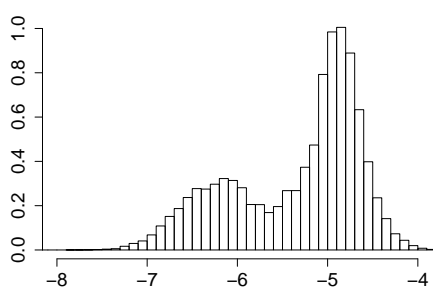

(k) $\phi^{\square} \square-\phi^{\{\emptyset\}}$

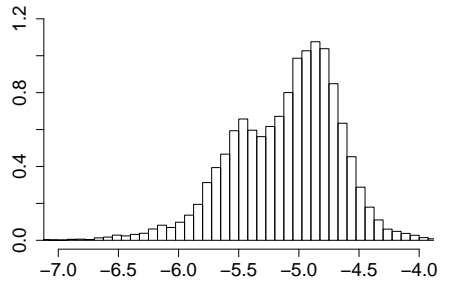

(c) $\phi \square-\phi^{\{\emptyset\}}$

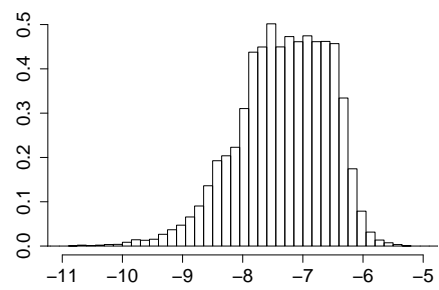

(f) $\phi \Phi^{-\phi^{\{\emptyset\}}}$

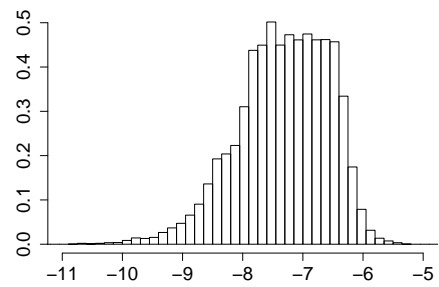

(i) $\phi \square-\phi^{\{\emptyset\}}$

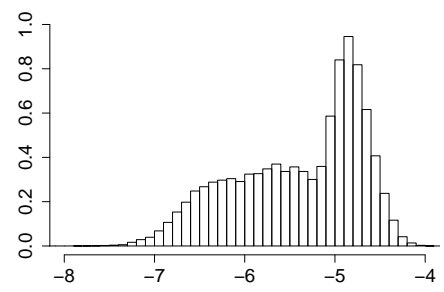

(l) $\phi \square-\phi^{\{\emptyset\}}$

Figure 12: Red deer example: Probability histograms for the posterior simulated values for $\phi^{\Lambda}-\phi^{\{\emptyset\}}$ for twelve values of $\Lambda$.

To investigate the fit of the likelihood to the data we did the following small experiment, similar to what we did for the $2 \times 2$ clique MRF example. For three randomly chosen realisations of $M, z, x_{b}$ and $\kappa$ we simulated from the likelihood three realisations of $x$. The results are shown in Figure 13, and we see that these realisation, at least visually, seem to have the same spatial dependence structure as the data in Figure 11.

Figure 14 shows posterior probability histograms for the prior-parameters $\eta, p_{\star}$ and $\sigma_{\varphi^{2}}$. 



Figure 13: Red deer example: Simulations for three parameter realisation.



(a) $\eta(0.739,2.357)$

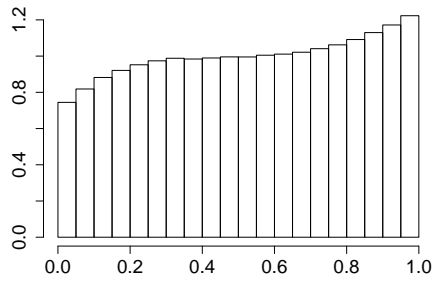

(b) $p_{\star}(0.034,0.978)$

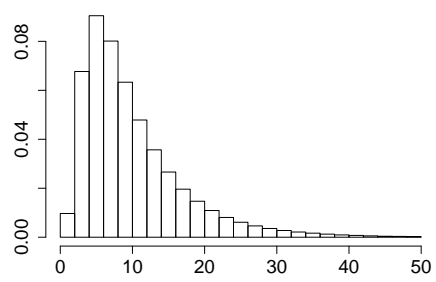

(c) $\sigma_{\varphi^{2}}(2.143,29.080)$

Figure 14: Red deer example: Prior-parameters with $95 \%$ credible interval.

The median of $\eta$ is in this example 1.255. The median of $p_{\star}$ is 0.538 , and as we can see from the estimated posterior distribution of $p_{\star}$ we slightly prefer models where interactions of order higher than two are turned on. The median of $\sigma_{\varphi^{2}}$ is 8.058 for this example. Lastly the estimated posterior distributions of the covariate parameters with $95 \%$ credible interval are given i Figure 15, As we can see all of these have credible intervals that does not include zero, supporting the need to include them in the model.

\section{Closing remarks}

In this paper we propose a prior distribution for the dependence structure of a stationary binary MRF. Our prior is formulated in three levels. First we define a prior for the neighbourhood system. Next, given the neighbourhood system, and thereby the set of cliques, we formulate a prior for the parametric form of the potential functions. In particular this includes a prior for whether higher-order interactions should be included in the MRF, and non-zero prior probabilities are assigned for the event that groups of parameters have exactly the same value. The latter serves both as a way to reduce the effective number of parameters and to allow properties like symmetry and isotropy. Lastly, given the neighbourhood 


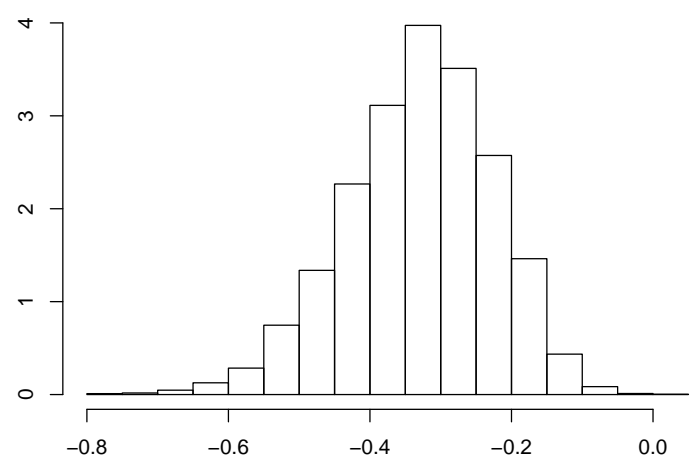

(a) $\kappa_{1}(-0.549,-0.146)$

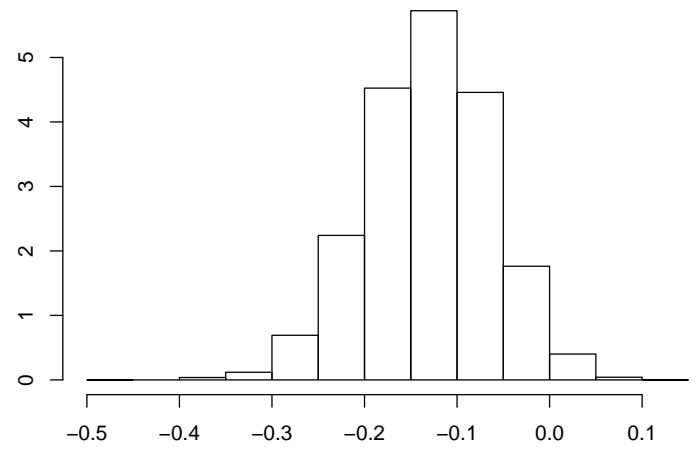

(c) $\kappa_{3}(-0.266,-0.002)$

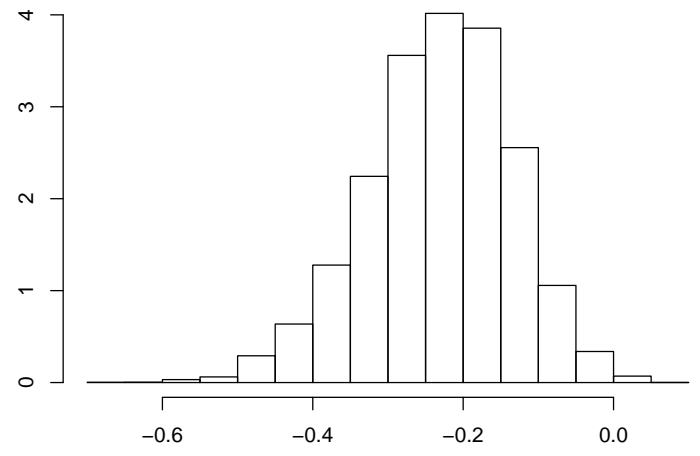

(b) $\kappa_{2}(-0.441,-0.058)$

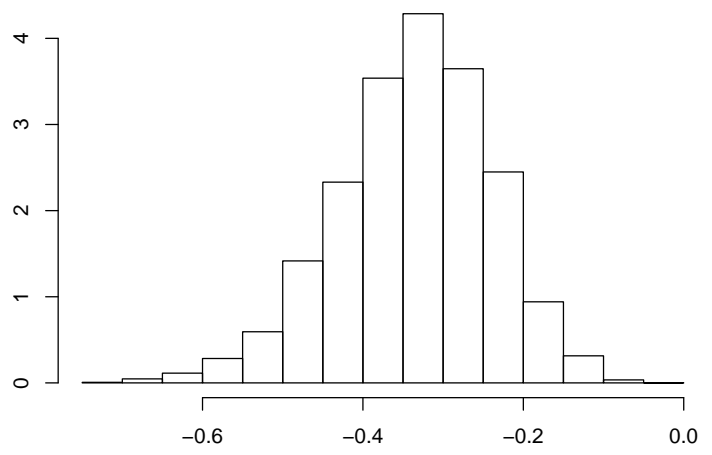

(d) $\kappa_{4}(-0.546,-0.164)$

Figure 15: Red deer example: Estimated marginal posterior distributions for the parameters of the covariates. Estimated $95 \%$ credible interval is given for each parameter.

system and parametric potential functions we define a prior for the parameter values. To our knowledge this is the first time a prior is formulated for all three levels in the dependency structure specification of an MRF. In particular it is thereby the first attempt to learn the neighbourhood system of an MRF from data via a fully Bayesian approach.

To sample from the resulting posterior distribution we adopt an RJMCMC approach, and in particular we formulate proposal distributions that can be used in such an algorithm. To cope with the computationally intractable normalising constant we adopt an auxiliary variable algorithm when computing acceptance probabilities, similar to what is done in Everitt (2012).

We present three examples of data in this paper. Two examples with simulated data, one from the Ising model and one from an MRF with $2 \times 2$ maximal cliques. Both the dependency 
structure and parameter values in these two examples are reasonably well recovered. The last example is a data set of presence/absence of red deer in a region in Scotland. The results show a clear spatial dependency within the data. In particular the spatial dependency involves higher order interaction, which demonstrates the importance of exploring higher order interaction MRFs also for data sets where the spatial dependency is week. Clearly more experience with our proposed prior is needed, both on synthetic and real world data sets.

In the current article we restrict ourselves to consider only binary data. However a generalisation to the discrete case is straightforward. The bottleneck of this approach is clearly computation time, as we need to simulate an MCMC sample from the likelihood for each proposed change in the parameter vector. A possible strategy to reduce this problem would be to replace the MRF with a partially ordered Markov model (POMM), see Qian and Titterington (1991), Cressie and Davidson (1998) and Toftaker (2013). The POMM is known to be computationally superior to MRF, and we are currently exploring such an option.

Another important direction for future research is to improve the proposal distributions in order to obtain better convergence and mixing of the MCMC algorithm, which in turn also would improve the overall computation time. We believe that better proposal distributions would help the MCMC algorithm to converge also for more complex data sets than what we have presented here. With our current proposal distributions, we have in particular found it hard to get satisfactory convergence and mixing properties for data sets with strong spatial dependence. One improvement that we believe would help the convergence is in how to propose new parameter values when adding or removing a parameter. For example, when proposing to add a new parameter in our current algorithm we just propose a new value for this new parameter and keep all the other parameter values unchanged, except for the sum-to-zero adjustment. A better alternative would be to propose a change also in the other parameter values so that the new model is closer, in some sense, to the current model. Fixing the value of the potential new parameter, one possibility would be to define the potential new values of the remaining parameters by defining the potential new model as a least squares approximation of the current model. This strategy has strong similarities with an approximation technique used in Austad (2011), but the results there can not directly be 
used in our setting because we have adopted a partition of $M$.

\section{Supporting Information}

Additional Supporting Information may be found in the online version of this article:

Section S.1: Details for the MCMC sampling algorithm.

Section S.2: Investigating the modality in the red deer example.

\section{References}

Arnesen, P. and Tjelmeland, H. (2015). "Fully Bayesian binary Markov random field models: Prior specification and posterior simulation." ArXiv e-prints, arXiv:1501.04419v1.

Augustin, N. H., Mugglestone, M. A., and Buckland, S. T. (1996). "An autologistic model for the spatial distribution of wildlife." Journal of Applied Ecology, 33, 339-347.

Austad, H. M. (2011). "Approximations of Binary Markov Random Fields." Ph.D. thesis, Norwegian University of Science and Technology. Thesis number 292:2011. Available from http://urn.kb.se/resolve?urn=urn:nbn:no:ntnu:diva-14922.

Besag, J. (1974). "Spatial interaction and the statistical analysis of lattice systems." Journal of the Royal Statistical Society. Series B (Methodological), 36, 192-236.

- (1986). "On the statistic analysis of dirty pictures." Journal of the Royal Statistical Society. Series B (Methodological), 48, 259-302.

Buckland, S. T. and Elston, D. A. (1993). "Empirical models for the spatial distribution of wildlife." Journal of Applied Ecology, 30, 478-495.

Caimo, A. and Friel, N. (2011). "Bayesian inference for exponential random graph models." Social Networks, 33, 41-55. 
Celeux, G., Hurn, M. A., and C. P. Robert, C. (2000). "Computational and inferential difficulties with mixture posterior distributions." 95, 957-970.

Clifford, P. (1990). "Markov random fields in statistics." In Disorder in Physical Systems, A Volume in Honour of John M.Hammersley, eds. G. Grimmett and D. J. Welsh. Oxford University Press.

Cressie, N. and Davidson, J. L. (1998). "Image analysis with partially ordered Markov models." Computational Statistics and Data Analysis, 29, 1-26.

Cressie, N. A. (1993). Statistics for Spatial Data. 2nd ed. New York: John Wiley.

Dellaportas, P. and Forster, J. J. (1999). "Markov chain Monte Carlo model determination for hierarchical and graphical log-linear models." Biometrika, 86, 615-633.

Descombes, X., Mangin, J., Pechersky, E., and Sigelle, M. (1995). "Fine structures preserving Markov model for image processing." In Proc. 9th SCIA 95, Uppsala, Sweden, 349-356.

Everitt, R. G. (2012). "Bayesian parameter estimation for latent Markov random fields and social networks." Journal of Computational and Graphical Statistics, 21, 940-960.

Friel, N., Pettitt, A. N., Reeves, R., and Wit, E. (2009). "Bayesian inference in hidden Markov random fields for binary data defined on large lattices." 18, 243-261.

Friel, N. and Rue, H. (2007). "Recursive computing and simulation-free inference for general factorizable models." Biometrika, 94, 661-672.

Geyer, C. J. and Thompson, E. A. (1992). "Constrained Monte Carlo maximum likelihood for dependent data." Journal of the Royal Statistical Society. Series B (Methodological), $54,657-699$.

Grabisch, M., Marichal, L.-L., and Roubens, M. (2000). "Equivalent representation of set function." Mathematics of operations reasearch, 25, 157-178.

Green, P. J. (1995). "Reversible jump MCMC computation and Bayesian model determination." Biometrika, 82, 711-732. 
Hammer, P. and Holzman, R. (1992). "Approximations of pseudo-boolean functions; application to game theory." Methods and models of operations research, 36, 3-21.

Heikkinen, J. and Högmander, H. (1994). "Fully Bayesian approach to image restoration with an application in biogeography." Applied Statistics, 43, 569-582.

Higdon, D. M., Bowsher, J. E., Johnsen, V. E., Turkington, T. G., Gilland, D. R., and Jaszczak, R. J. (1997). "Fully Bayesian estimation of Gibbs hyperparameters for emission computed tomography data." IEEE Transactions on medical imaging, 16, 516-526.

Hurn, M., Husby, O., and Rue, H. (2003). "A tutorial on image analysis." In Spatial Statistics and Computational Methods, ed. J. Møller, vol. 173 of Lecture Notes in Statistics, 87-141. Springer Verlag.

Kindermann, R. and Snell, J. L. (1980). Markov Random Fields and Their Applications. Providence, Rhode Island: American Mathematical Society.

Lauritzen, S. (1996). Graphical Models. Oxford: Clarendon Press.

Madigan, D., York, J., and Allard, D. (1995). "Bayesian graphical models for discrete data." International Statistical Review, 63, 215-232.

Massam, H., Liu, J., and Dobra, A. (2009). "A conjugate prior for discrete hierarchical log-linear models." The Annals of Statistics, 37, 3431-3467.

McGrory, C. A., Pettitt, A. N., Reeves, R., Griffin, M., and Dwyer, M. (2012). "Variational Bayes and the reduced dependence approximation for the autologistic model on an irregular grid with applications." Journal of Computational and Graphical Statistics, 21, 781-796.

Møller, J., Pettitt, A., Reeves, R., and Berthelsen, K. (2006). "An efficient Markov chain Monte Carlo method for distributions with intractable normalising constants." Biometrika, $93,451-458$.

Murray, I., Ghahramani, Z., and MacKay, D. (2006). "MCMC for doubly-intractable distributions." In Proceedings of the Twenty-Second Conference Annual Conference on Uncertainty in Artificial Intelligence (UAI-06), 359-366. Arlington, Virginia: AUAI Press. 
Propp, J. G. and Wilson, D. B. (1996). "Exact sampling with coupled Markov chains and applications to statistical mechanics." Random Structures \& Algorithms, 9, 223-252.

Qian, W. and Titterington, D. M. (1991). "Multidimensional Markov chain models for image textures." Journal of the Royal Statistical Society. Series B (Methodological), 53, 661-674.

Ronald L. Graham, Donald E. Knuth, O. P. (1988). Concrete Mathematics. 2nd ed. Reading MA: Addison-Wesley.

Rydén, T. and Titterington, D. M. (1998). "Computational Bayesian analysis of hidden Markov models." Journal of Computational and Graphical Statistics, 7, 194-211.

Spiegelhalter, D. J., Dawid, A. P., Lauritzen, S. L., and Cowell, R. G. (1993). "Bayesian analysis in expert systems." Statistical Science, 8, 219-247.

Tjelmeland, H. and Austad, H. M. (2012). "Exact and approximate recursive calculations for binary Markov random fields defined on graphs." Journal of Computational and Graphical Statistics, 21, 758-780.

Tjelmeland, H. and Besag, J. (1998). "Markov random fields with higher order interactions." Scandinavian Journal of Statistics, 25, 415-433.

Toftaker, H. (2013). "Modelling and parameter estimation for discrete random fields and spatial point processes." Ph.D. thesis, Norwegian University of Science and Technology. Thesis number 200:2013. Available from http://ntnu.divaportal.org/smash/record.jsf?pid=diva2:662513. 


\title{
Supplementary materials to the paper: Prior specification of neighbourhood and interaction structure in binary Markov random fields
}

\author{
Petter Arnesen and HÅon TJelmeland \\ Department of Mathematical Sciences, Norwegian University of Science and Technology
}

\section{S.1 Details for the MCMC sampling algorithm}

In this section we provide details of the proposal distributions that we use when sampling from the posterior distribution

$$
p(z, M, \theta \mid x) \propto p(x \mid z, M) p(z \mid M, \theta) p(M \mid \theta) p(\theta)
$$

where $p(x \mid z, M)$ is the likelihood MRF and $p(z \mid M, \theta), p(M \mid \theta)$ and $p(\theta)$ are the priors defined in Section 5 in the paper. All of the proposal distributions are also briefly discussed in Section 6 in the paper. The proposals given in Sections $\underline{\mathbf{S . 1 . 3}}$ and $\mathbf{S . 1 . 6}$ are transdimensional proposals, resulting in reversible jump Markov chain Monte Carlo (RJMCMC) updates. Note that all proposals cannot be applied to all states, or that some of these proposals could result in non-reversible states. For these cases we simply regard the proposal as a reject. In this section we use $S, S^{\star}$ and $S^{\star \star}$ to denote specific elements of the partition $\mathscr{S}$.

\section{S.1.1 Random walk for parameter updates}

Assume the current state to be $z=\left\{\left(S, \varphi_{S}\right) ; S \in \mathscr{S}\right\}$. The first proposal is simply a random walk proposal for the $\varphi$ parameters, but correcting the update so that the parameter values still sum to zero. We do this by first uniformly at random selecting one of the $\varphi$ parameters, $\varphi_{S^{\star}}$ for $S^{\star} \in \mathscr{S}$ say, and add to it a random change $\varepsilon \sim N\left(0, \sigma_{w}^{2}\right)$, where $\sigma_{w}$ is an algorithmic tuning parameter. Next, for the proposed new parameter values to comply with the sumto-zero constraint, we subtract the same value from all the parameters. The potential new state $\tilde{z}$ becomes

$$
\tilde{z}=\left\{\left(S, \varphi_{S}-\frac{\varepsilon}{\operatorname{card}(\mathscr{S})}\right) ; S \in \mathscr{S} \backslash\left\{S^{\star}\right\}\right\} \cup\left\{\left(S^{\star}, \varphi_{S^{\star}}+\varepsilon-\frac{\varepsilon}{\operatorname{card}(\mathscr{S})}\right)\right\} .
$$




\section{S.1.2 Switching cell for one clique type}

Assume again the current state to be $z=\left\{\left(S, \varphi_{S}\right) ; S \in \mathscr{S}\right\}$. Note that this proposal is not possible if the number of cells equals one or all the cells contains only one clique type each. To generate a potential new state we first sample a pair of distinct cells $S^{\star}, S^{\star \star} \in \mathscr{S}$ from the distribution

$$
q\left(S^{\star}, S^{\star \star}\right) \propto \begin{cases}\exp \left\{-\left(\varphi_{S^{\star}}-\varphi_{S^{\star \star}}\right)^{2}\right\} & \text { if } S^{\star} \neq S^{\star \star} \text { and } \operatorname{card}\left(S^{\star}\right)>1, \\ 0 & \text { otherwise. }\end{cases}
$$

Thus, we have that the number of clique types in $S^{\star}$ is strictly larger than one, and pairs $S^{\star}, S^{\star \star}$ where the corresponding parameter values are similar have a higher probability to be sampled. Given $S^{\star}$ and $S^{\star \star}$ the potential new state is formed by drawing at random a clique type $\Lambda \in S^{\star}$ and moving $\Lambda$ over to $S^{\star \star}$. The potential new state thereby becomes

$$
\tilde{z}=\left\{\left(S, \varphi_{S}\right) ; S \in \mathscr{S} \backslash\left\{S^{\star}, S^{\star \star}\right\}\right\} \cup\left\{\left(S^{\star} \backslash\{\Lambda\}, \varphi_{S^{\star}}\right),\left(S^{\star \star} \cup\{\Lambda\}, \varphi_{S^{\star \star}}\right)\right\} .
$$

\section{S.1.3 To propose a change in the partition}

Again let the current state be $z=\left\{\left(S, \varphi_{S}\right) ; S \in \mathscr{S}\right\}$. In this proposal we propose to increase or decrease the number of cells by one, with probability a half for each. We refer to these proposals as split and merge proposals, respectively. We start by explaining how to propose to increase the number of cells by one (split), before we explain the opposite move (merge). Note that the spilt proposal is not possible if the number of cells equals the number of clique types, and that the merge proposal is not possible if there is no cell with only one clique type or if $M=\{\{\emptyset\}\}$.

To propose to increase the number of cells by one we start by drawing uniformly at random one of the cells where the number of clique types in the cell are larger than one. Assuming cell $S^{\star}$ is sampled, we next uniformly at random draw one of the clique types in this cell, $\Lambda \in S^{\star}$ say, and use this to form a new cell including only this clique type, i.e. $\{\Lambda\}$. This new cell must be assigned an associated parameter value, $\varphi_{\{\Lambda\}}$, and to do this we add an $\varepsilon \sim \mathrm{N}\left(0, \sigma_{g}^{2}\right)$, where $\sigma_{g}^{2}$ is an algorithmic tuning parameter, to the old parameter value for $\Lambda, \varphi_{S^{\star}}$. Finally, for the potential new parameter values to comply with the sum-to-zero 
constraint, we subtract the same value from all the parameters, so that the potential new state becomes

$$
\begin{aligned}
\tilde{z}= & \left\{\left(S, \varphi_{S}-\frac{\varphi_{S}+\varepsilon}{\operatorname{card}(\mathscr{S})+1}\right) ; S \in \mathscr{S} \backslash\left\{S^{\star}\right\}\right\} \cup \\
& \left\{\left(S^{\star} \backslash\{\Lambda\}, \varphi_{S^{\star}}-\frac{\varphi_{S^{\star}}+\varepsilon}{\operatorname{card}(\mathscr{S})+1}\right),\left(\{\Lambda\}, \varphi_{S^{\star}}+\varepsilon-\frac{\varphi_{S^{\star}}+\varepsilon}{\operatorname{card}(\mathscr{S})+1}\right)\right\} .
\end{aligned}
$$

To propose to decrease the number of cells, we can only consider to merge a cell, $S^{\star}$ say, consisting of only one clique type, i.e. $\operatorname{card}\left(S^{\star}\right)=1$, into another cell, $S^{\star \star}$ say, in order for this proposal to conform with the split move specified above. We therefore first sample two distinct cells $S^{\star}, S^{\star \star} \in \mathscr{S}$ from the distribution

$$
q\left(S^{\star}, S^{\star \star}\right) \propto \begin{cases}\exp \left\{-\left(\varphi_{S^{\star}}-\varphi_{S^{\star \star}}\right)^{2}\right\} & \text { if } S^{\star} \neq S^{\star \star} \text { and } \operatorname{card}\left(S^{\star}\right)=1, \\ 0 & \text { otherwise. }\end{cases}
$$

Given $S^{\star}$ and $S^{\star \star}$ the potential new state is formed by first merging $S^{\star}$ into $S^{\star \star}$ and thereafter correcting all parameter values with the same amount to obtain a proposal that comply with the sum-to-zero constraint. The potential new state then becomes

$\tilde{z}=\left\{\left(S, \varphi_{S}+\frac{\varphi_{S^{\star}}}{\operatorname{card}(\mathscr{S})-1}\right) ; S \in \mathscr{S} \backslash\left\{S^{\star}, S^{\star \star}\right\}\right\} \cup\left\{\left(S^{\star} \cup S^{\star \star}, \varphi_{S^{\star \star}}+\frac{\varphi_{S^{\star}}}{\operatorname{card}(\mathscr{S})-1}\right)\right\}$.

The split and merge proposals give a change in the dimension of the parameter space, and in the acceptance probability for these RJMCMC steps we then need to include a Jacobi determinant. For the split and merge proposals specified above it is straightforward to show that the Jacobi determinants become $\frac{\operatorname{card}(\mathscr{S})}{\operatorname{card}(\mathscr{S})+1}$ and $\frac{\operatorname{card}(\mathscr{S})}{\operatorname{card}(\mathscr{S})-1}$, respectively.

\section{S.1.4 To propose a change in $M$ by replacing a clique type}

In this step we propose to replace one of the clique types that is on with another clique type. Note that this proposal is not possible if there are no clique types with $\tau(\Lambda)>1$, or if the proposed clique type either consists of two equal nodes or is equal to a clique type already in $M$, in both cases giving a non-reversible state. Assuming the current state to be $M$ and $z=\left\{\left(S, \varphi_{S}\right) ; S \in \mathscr{S}\right\}$, we start this proposal by uniformly drawing one of the clique types $\Lambda \in M$ where $\tau(\Lambda)>1$. In the following we let $S^{\star}$ denote the cell in $\mathscr{S}$ that contains $\Lambda$. Then consider an arbitrary element in $\Lambda, \lambda \in \Lambda$ say, and draw at random one of the 
elements in $\lambda,(i, j)$ say. We then generate a modified version of $\lambda$ by replacing $(i, j)$ with one of the first order neighbours of $(i, j)$. More formally, we first draw uniformly at random a $(k, l)$ from the set $\{(0,1),(1,0),(0, n-1),(m-1,0)\}$, and let the modified version of $\lambda$ be given by $\lambda^{\star}=(\lambda \backslash\{(i, j)\}) \cup\{(i, j) \oplus(k, l)\}$. A corresponding modified version of $\Lambda$ is then $\Lambda^{\star}=\left\{\lambda^{\star} \oplus(t, u) ;(t, u) \in \chi\right\}$. The potential new state is then generated by just replacing $\Lambda$ with $\Lambda^{\star}$ in the definition of $M$ and $z$. The potential new values of $M$ and $z$ becomes $\tilde{M}=(M \backslash\{\Lambda\}) \cup\left\{\Lambda^{\star}\right\}$ and $\tilde{z}=\left\{\left(S, \varphi_{S}\right) ; S \in \mathscr{S} \backslash\left\{S^{\star}\right\}\right\} \cup\left\{\left(\left(S^{\star} \backslash\{\Lambda\}\right) \cup\left\{\Lambda^{\star}\right\}, \varphi_{S^{\star}}\right)\right\}$.

\section{S.1.5 To propose a change in $M$ by adding/deleting a clique type}

Again let the current state be $M$ and $z=\left\{\left(S, \varphi_{S}\right) ; S \in \mathscr{S}\right\}$. In this proposal we either add a clique type to $M$ or delete a clique type from $M$, but keeping the same number of $\varphi$ parameters. For the adding proposal note that a clique type that is already in $M$ could be proposed or that the proposed clique type could consist of two equal nodes, in both cases giving a non-reversible state. Also, the deleting proposal is not possible if there are no cells with more than one clique type. First we draw at random whether to increase or decrease the number of clique types, with probability a half for each. In the following we first explain the increase proposal and thereafter the decrease proposal.

We now explain how we propose to increase the number of clique types, keeping the same number of $\varphi$ parameters. We start by drawing uniformly at random one of the existing clique types in $M, \Lambda$ say. A new clique type $\Lambda^{\star}$ is then generated in three steps. First we take out an arbitrary element of $\Lambda, \lambda$ say, and draw uniformly at random a node $(i, j)$ from the set of nodes in $\lambda$. Secondly we draw uniformly at random a $(k, l) \in \chi \backslash\{(0,0)\}$ from the distribution

$$
q(k, l) \propto \exp (-\rho(|k|+|l|)) \text { for }(k, l) \in \chi \backslash\{(0,0)\},
$$

where $\rho$ is an algorithmic tuning parameter. Given $(k, l)$ a new modified version of $\lambda, \lambda^{\star}$ is constructed by $\lambda^{\star}=\lambda \cup\{(i, j) \oplus(k, l)\}$. Thirdly, a modified version of $\Lambda, \Lambda^{\star}$ is constructed by $\Lambda^{\star}=\Lambda \cup\left\{\lambda^{\star} \oplus(t, u) ;(t, u) \in \chi\right\}$, and we generate the potential new value of $M$ by $\tilde{M}=M \cup\left\{\Lambda^{\star}\right\}$. To generate a corresponding potential new value for $z$ we first draw uniformly at random a cell $S^{\star}$ from all the cells in $\mathscr{S}$, and then let the potential new value 
of $z$ be given by $\tilde{z}=\left\{\left(S, \varphi_{S}\right) ; S \in \mathscr{S} \backslash\left\{S^{\star}\right\}\right\} \cup\left\{\left(S^{\star} \cup\left\{\Lambda^{\star}\right\}, \varphi_{S^{\star}}\right)\right\}$.

Next we explain the proposal we make when the number of clique types is to be decreased by one, but keeping the same number of $\varphi$ parameters. We start by uniformly drawing one of the clique types that belongs to a cell with more than one element. Let $\Lambda$ denote the clique type we draw and let $S^{\star}$ denote the cell that contains $\Lambda$. The potential new state is then generated simply by removing $\Lambda$ from the current state, so that the potential new values for $M$ and $z$ become $\tilde{M}=M \backslash\{\Lambda\}$ and $z^{\star}=\left\{\left(S, \varphi_{S}\right) ; S \in \mathscr{S} \backslash\left\{S^{\star}\right\}\right\} \cup\left\{\left(S^{\star} \backslash\{\Lambda\}, \varphi_{S^{\star}}\right)\right\}$, respectively.

\section{S.1.6 To propose a change in $M$ and in the partition}

Let again the current state be $M$ and $z=\left\{\left(S, \varphi_{S}\right) ; S \in \mathscr{S}\right\}$. In this proposal we either add a clique type to $M$ or delete a clique type from $M$, and increase or decrease the number of $\varphi$ parameters correspondingly. For the adding proposal note again that a clique type that is already in $M$ could be proposed or that the proposed clique type could consist of two equal nodes, in both cases giving a non-reversible state. Also, the deleting proposal is not possible if there are no cells with only one clique type or $M=\{\{\emptyset\}\}$. We first draw at random which of these two possible proposals to perform, with probability a half for each. In the following we start by explaining the increasing proposal, and thereafter move on to the decreasing proposal.

We start by generating a $\Lambda^{\star}$ in exactly the same way as we did for the increase proposal in Section S.1.5. Next, we form a new cell containing only $\Lambda^{\star}$. To generate the associated parameter value $\varphi_{\left\{\Lambda^{\star}\right\}}$ we follow a strategy similar to what we did in Section S.1.3. We first add an $\varepsilon \sim N\left(0, \sigma_{c}^{2}\right)$, where $\sigma_{c}$ is an algorithmic tuning parameter, to the current value $\phi^{\Lambda^{\star}}$, and thereafter subtract the same value from all the $\varphi$ parameters to get a proposal that comply with the sum-to-zero constraint. The proposed new values for $M$ and $z$ then becomes $\tilde{M}=M \cup\left\{\Lambda^{\star}\right\}$ and

$$
\tilde{z}=\left\{\left(S, \varphi_{S}-\frac{\phi^{\Lambda^{\star}}+\varepsilon}{\operatorname{card}(\mathscr{S})+1}\right) ; S \in \mathscr{S}\right\} \cup\left\{\left(\left\{\Lambda^{\star}\right\}, \phi^{\Lambda^{\star}}+\varepsilon-\frac{\phi^{\Lambda^{\star}}+\varepsilon}{\operatorname{card}(\mathscr{S})+1}\right)\right\},
$$

respectively.

Next we explain how we generate a proposal where the number of clique types is decreased 
by one, and the number of $\varphi$ parameters is correspondingly reduced. We start by drawing uniformly at random one of the clique types that is the only member of a cell. Let $\Lambda$ denote the one we sample and let $S^{\star}=\{\Lambda\}$ denote the corresponding cell. This clique type and its corresponding cell is now simply deleted from the current state resulting in the proposed values $\tilde{M}=M \backslash\{\Lambda\}$ and

$$
\tilde{z}=\left\{\left(S, \varphi_{S}+\frac{\varphi_{S^{\star}}}{\operatorname{card}(\mathscr{S})-1}\right) ; S \in \mathscr{S} \backslash\left\{S^{\star}\right\}\right\},
$$

where we again has subtracted the same value from all the $\varphi$ parameters to comply with the sum-to-zero constraint. As in Section $\underline{S .1 .3}$ it is straightforward to show that the Ja-

cobi determinants for the increase and decrease proposals become $\frac{\operatorname{card}(\mathscr{S})}{\operatorname{card}(\mathscr{S})+1} \operatorname{and} \frac{\operatorname{card}(\mathscr{S})}{\operatorname{card}(\mathscr{S})-1}$ respectively.

\section{S.1.7 Proposal for the model parameters}

In total we have three model parameters that we assign prior distributions to, namely $\eta$, $p_{\star}$ and $\sigma_{\varphi}^{2}$. We make one proposal for each of these parameters for every iteration of the sampling algorithm. Firstly we update $\eta$ by proposing a zero mean Gaussian change with standard deviation $\sigma_{\eta}$. Secondly we propose a new value for $p_{\star}$ simply by sampling a new value from a uniform distribution on the interval $(0,1)$. For the last parameter $\sigma_{\varphi}^{2}$ we propose a zero mean Gaussian change with standard deviation $\sigma_{\sigma_{\varphi}}$.

\section{S.2 The multi-modality in the red deer example}

In this section we provide a more detailed investigation of the multi-modality in the posterior distributions for $\phi^{\Lambda}-\phi^{\{\emptyset\}}$ shown in Figure 12 in Section 7.3. As mentioned in the paper the modes can be explained by first splitting the posterior samples into two groups, depending on the value of $M$. The first group is all samples where $M$ takes the aposteriori most probable value $M_{\max }=\{\{\emptyset\}, \square, \square, 曰$, ६, 虫, 由 $\}$, and the second group is the rest of the samples, i.e. samples where $M \neq M_{\max }$. From Table 3 we see that the posterior probabilities of these two groups are 0.284 and 0.716 , respectively. Making individual histograms for the $\phi$ values in these two groups give the results shown in the second and third rows in Figures S.2.1 S.2.4, 
which can be compared with the corresponding complete histogram in the first row. As we can see the results for $M_{\max }$ are unimodal, whereas for $M \neq M_{\max }$ some of the histograms are still bimodal, see in particular the histograms for $\square$, 日, ६, ${ }^{\square}$ and ${ }_{\square}$. This bimodality can be explained by splitting the realisations in the group $M \neq M_{\max }$ into two subgroups, one for realisations of $\phi^{\Lambda}$ where $\Lambda$ is on, and one for realisations of $\phi^{\Lambda}$ where $\Lambda$ is off. The results for $\phi^{\Lambda}$ under this split is shown in the forth and fifth rows in Figures S.2.1 S.2.4. Note however that this spilt results in empty or almost empty sets of realisations of $\phi^{\Lambda}$ for some $\Lambda$, in which case we do not make a histogram (denoted NA in the figures). 


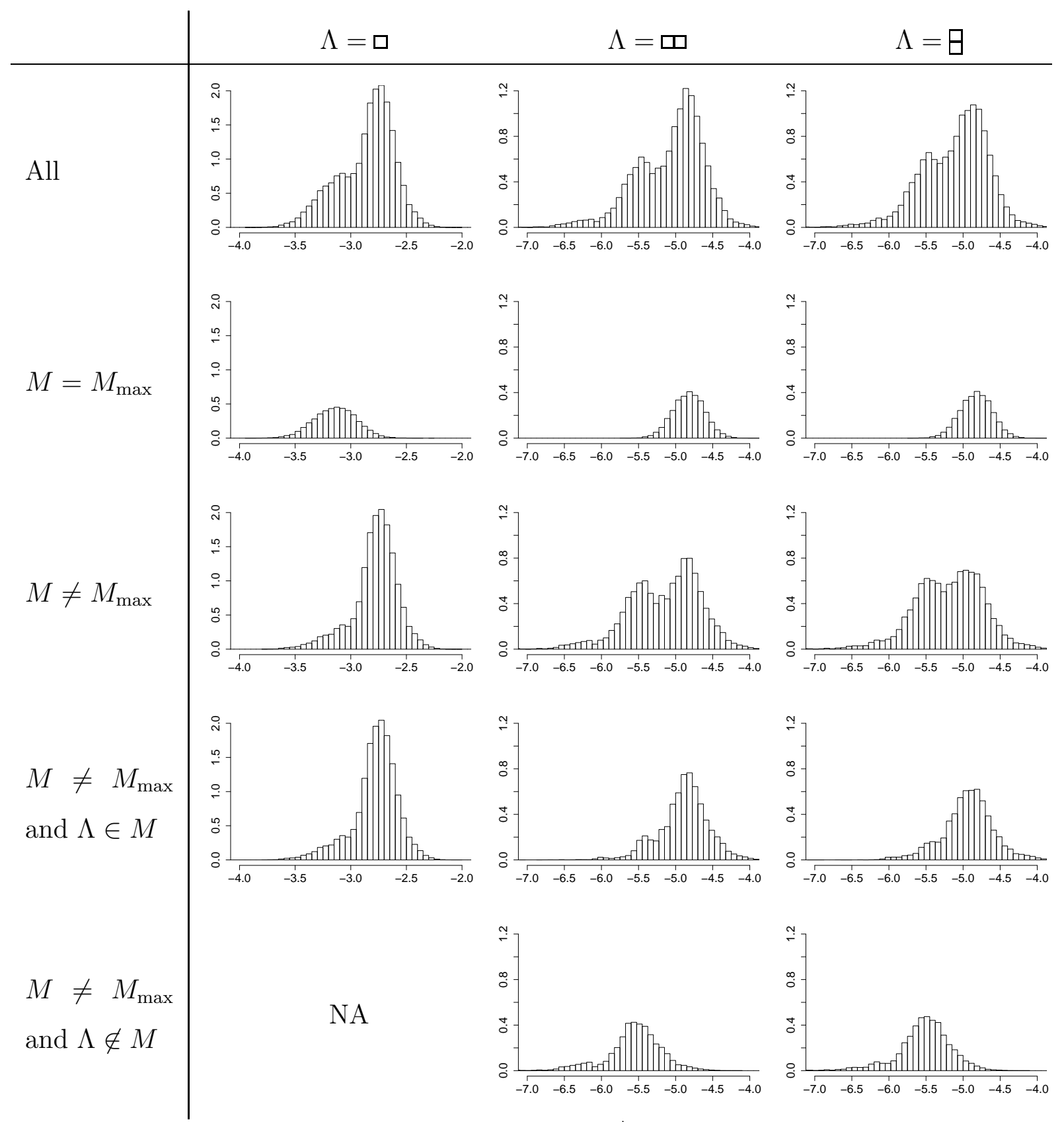

Figure S.2.1: Red deer example: Histograms for $\phi^{\Lambda}$ for some $\Lambda \in L$ for different subgroups of the posterior samples. Results for different clique types $\Lambda$ in each column, and different subgroups in each row. The conditions given in the first column are defining the different subgroups. 


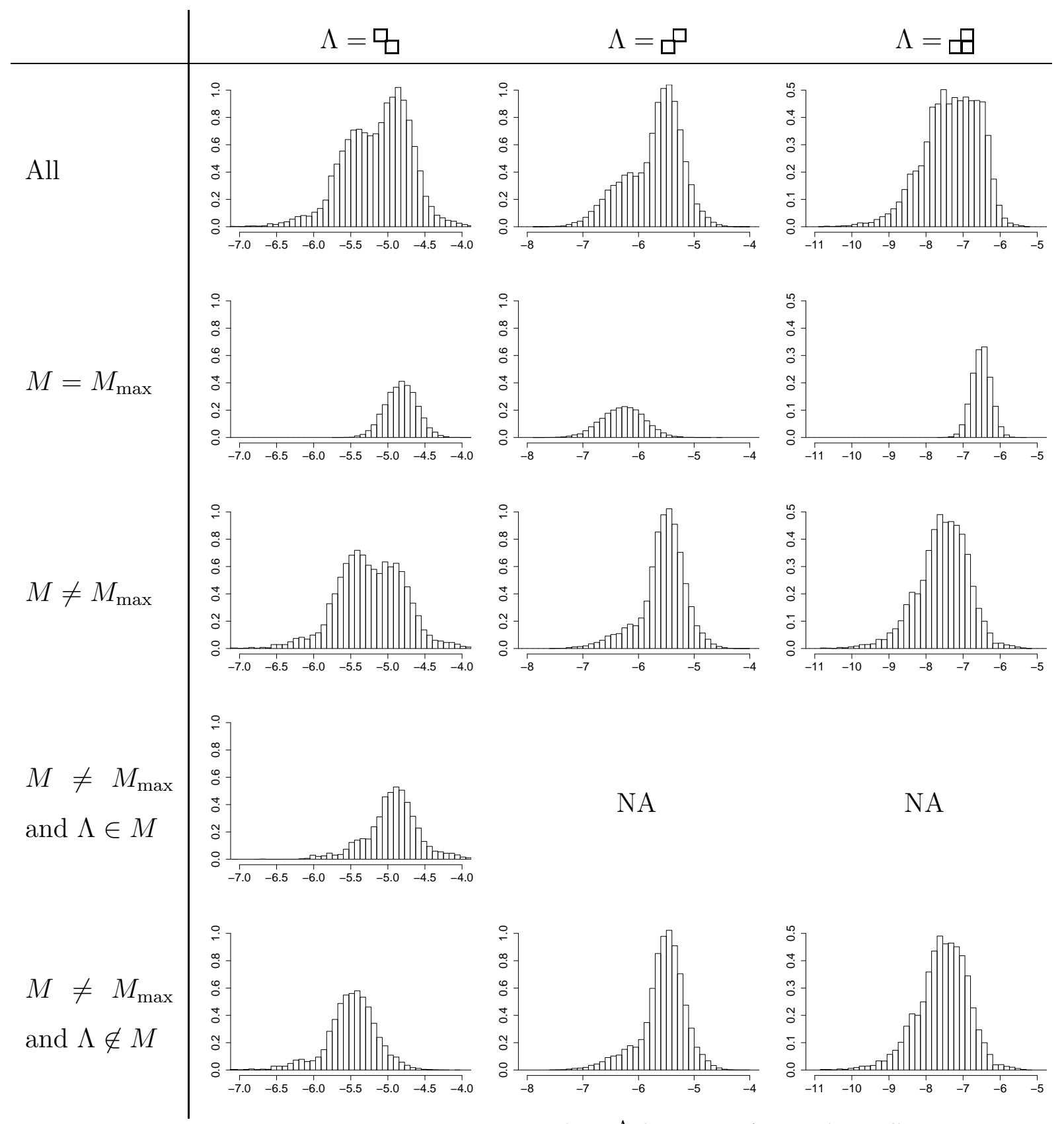

Figure S.2.2: Red deer example: Histograms for $\phi^{\Lambda}$ for some $\Lambda \in L$ for different subgroups of the posterior samples. Results for different clique types $\Lambda$ in each column, and different subgroups in each row. The conditions given in the first column are defining the different subgroups. 


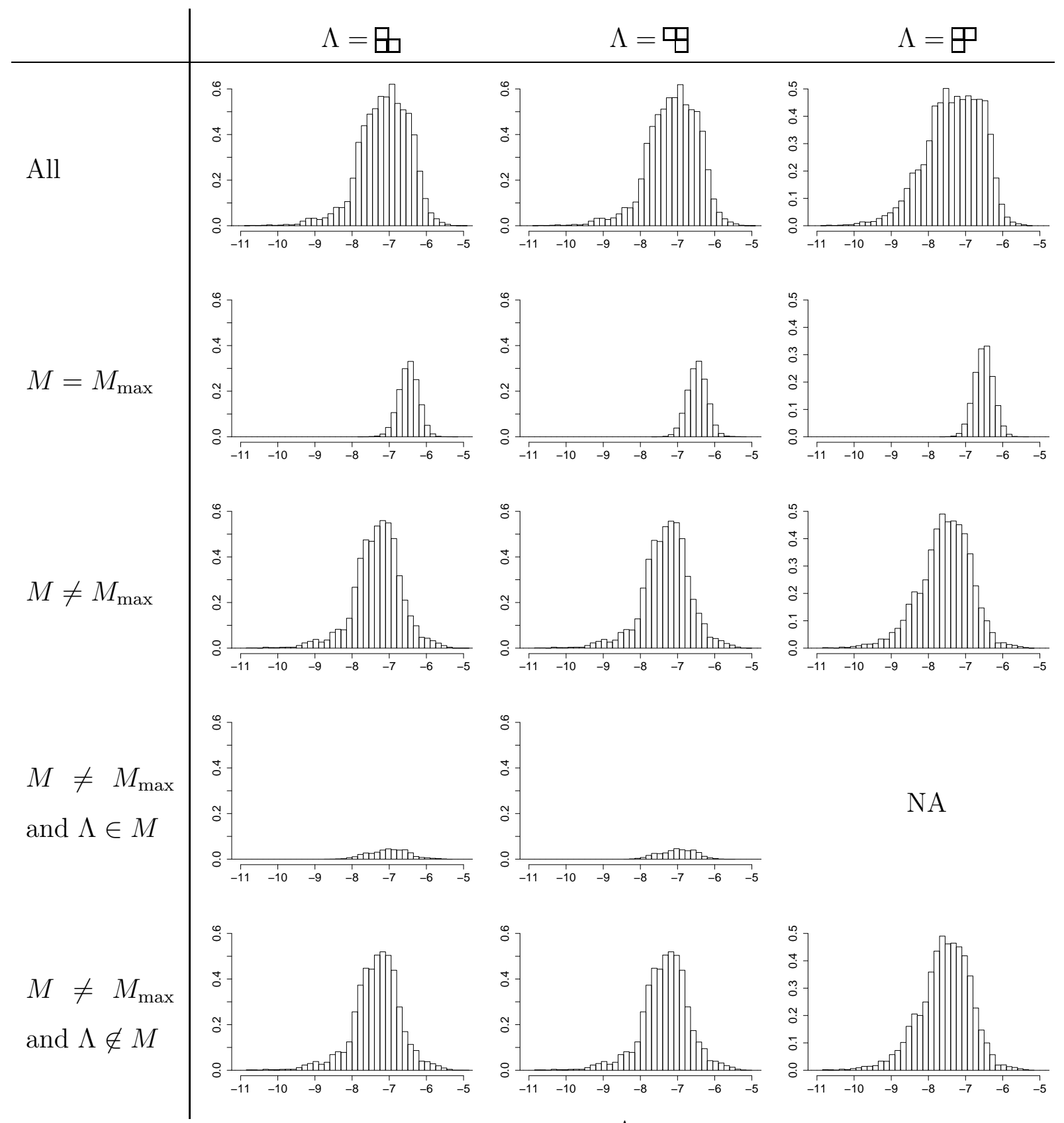

Figure S.2.3: Red deer example: Histograms for $\phi^{\Lambda}$ for some $\Lambda \in L$ for different subgroups of the posterior samples. Results for different clique types $\Lambda$ in each column, and different subgroups in each row. The conditions given in the first column are defining the different subgroups. 


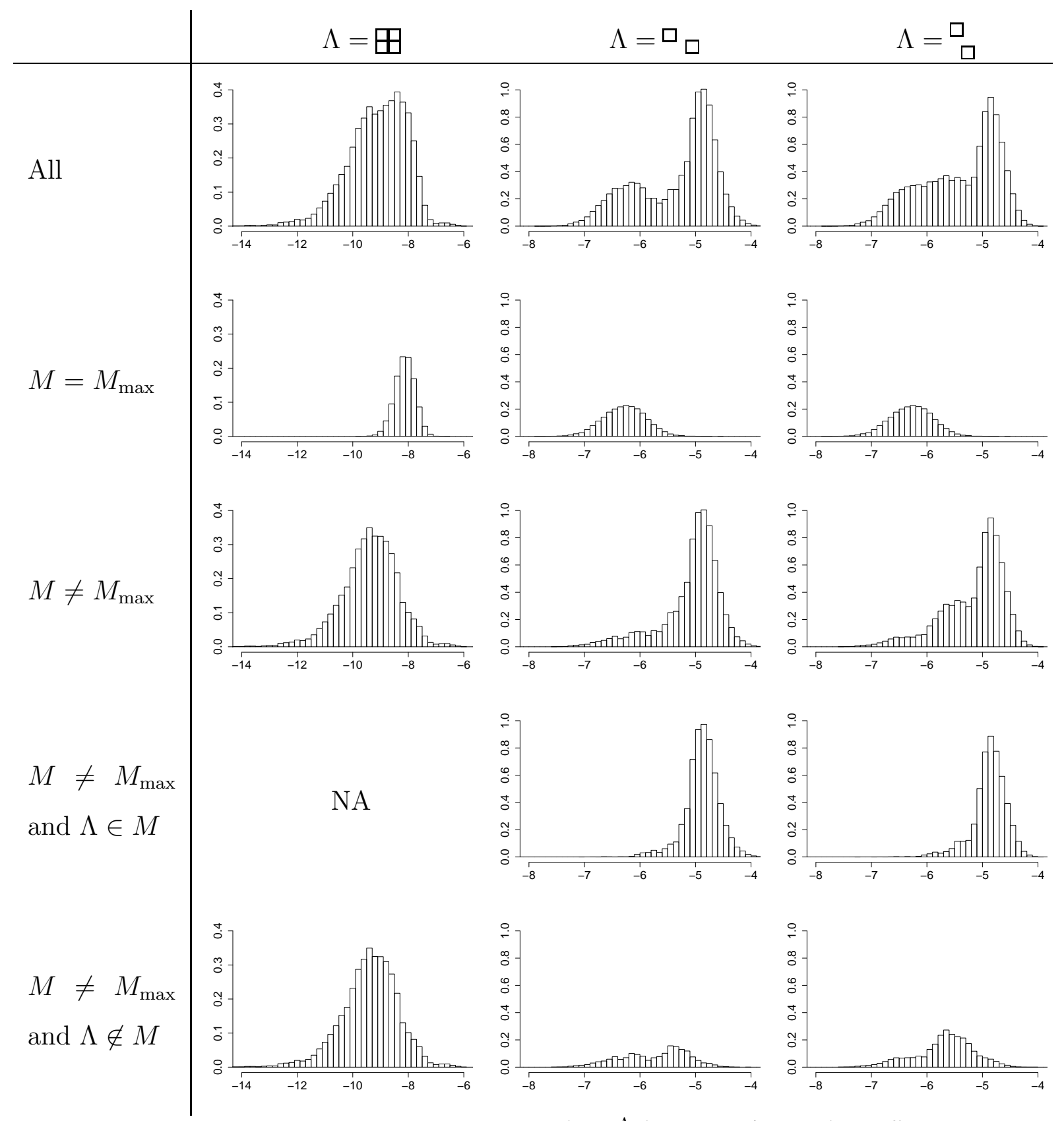

Figure S.2.4: Red deer example: Histograms for $\phi^{\Lambda}$ for some $\Lambda \in L$ for different subgroups of the posterior samples. Results for different clique types $\Lambda$ in each column, and different subgroups in each row. The conditions given in the first column are defining the different subgroups. 